\title{
Postprint:
}

\section{Experiments on Transonic Limit-Cycle-Flutter of a flexible swept Wing}

\author{
Günter Schewe \& Holger Mai \\ DLR-Institute of Aeroelasticity, Göttingen, Germany
}

The aeroelastic behavior of wing models is nonlinear particularly in the transonic speed range. The interaction between aerodynamic and structural forces can lead to the occurrence of Limit-Cycle Oscillations (LCOs). If in addition the wing model is flexible and backward swept, the kinematic coupling between bending and torsion makes the situation even more complex.

In the research project "Aerostabil" such a wing was investigated, which was equipped with pressure transducers in three sections and accelerometers. The experiments were performed in the adaptive test section of the transonic wind tunnel TWG in Göttingen. Already Dietz et al. (2003) have reported about experimental details and preliminary results. Based on these data Bendiksen (2008) studied numerically LCO-flutter behavior using a very similar, theoretical model (G-wing) and Stickan et al.(2014) used the original data as a LCO flutter test case. The influence of flexibility on the steady aerodynamics of the wing was described in Schewe $\&$ Mai (2018). In this paper now the flutter experiments with the same flexible model were analysed systematically in the transonic range $0.84<\mathrm{Ma}<0.89$ and for six angles of attack from $1.46^{\circ}$ to $2.7^{\circ}$. Maps of stability, LCO amplitudes and instantaneous pressure distributions are presented. It was found that unstable regions are islands, whose extent depends on the angle of attack. A LCO test case, already treated in the literature is examined in more detail. The analysis of the time functions showed that during LCO-flutter the motion induced aerodynamic sectional lift forces particularly in the outer wing are asymmetric and thus acting as amplitude limiter. The reason for the asymmetry lies in the shock/boundary layer interaction. The test case, containing the stages of built-up and the transition to the limit cycle provides an excellent opportunity for improving our knowledge about LCOs and for code validation purposes.

Keywords: Transonic Flow; Limit Cycle Oscillations; Transonic Flutter; flexible swept Wing; LCO Flutter; Influence of Flexibility 
Schewe, Günter, and Mai, Holger. "Experiments on transonic limit-cycle-flutter of a flexible swept wing." Journal of Fluids and Structures 84 (2019): pp. 153-170. http://doi.org/10.1016/j.jfluidstructs.2018.07.005

\section{Introduction}

The aeroelastic behavior of a nominally stiff wing model is nonlinear particularly in the transonic speed range. From the aerodynamic side the interaction between the shock dynamic, the boundary layer and its separation is a significant source of nonlinearity. Even the interplay between a linear structure and nonlinear aerodynamic forces leads to the occurrence of several manifestations of Limit-Cycle Oscillations (LCOs). In case of a flexible and backward swept wing the nonlinear behavior is made more complex by the kinematic coupling between bending and torsion leading to a washout effect. Thus the static bending and torsional deformations depending on the airloads can have drastic consequences on the flutter behavior. As mentioned in Schewe \& Mai (2018) already Jordan (1946) predicted the possibility of one degree of freedom flutter of a backward swept wing as consequence of the kinematic coupling effects between bending and torsion (se also Meier, 2010).

An early wind tunnel test with flexible models was performed in a German/French cooperation called "Aeroelastic Model Program (AMP)". One of two models was dynamically scaled especially for flutter tests. (Zingel et al. (1991), Arnold et al.(2009)). Figure 1 shows an unpublished time trace of a wing tip accelerometer taken at the onset of flutter at high transonic Mach number. Based on our present experience regarding flutter experiments we can conclude that the transition to a LCO is not far away, indicated by the small build-up rate of the exponential increasing wing tip oscillations.

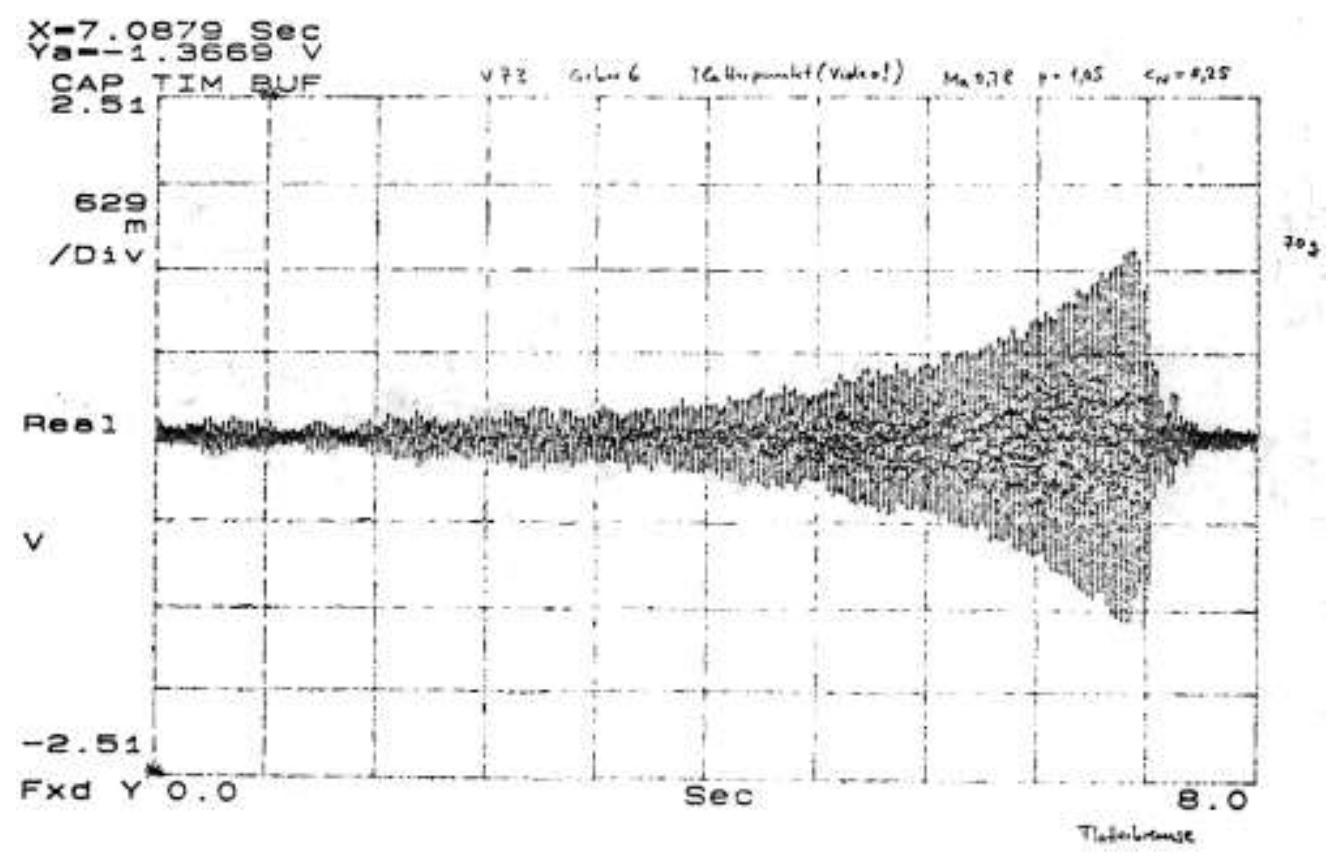

Figure 1. Flutter onset of the AMP-wing (Ma $=0.78$, normal force coefficient $\mathrm{C}_{\mathrm{N}}=0.25$, stagnation pressure $\mathrm{p}_{0}=1.05$ bar) taken in the $S 2$ wind tunnel in Modane (1991). As usual at that time, the oscillations were stopped mechanically.

A review of the unsteady transonic aerodynamics and aeroelasticity was performed by Tijdeman \& Seebass (1980). More recently Bendiksen (2011) presented a review focused on theoretical and CFD aspects. Regarding experimental studies there are only a few, which deal with LCO behavior of high-aspect-ratio elastic wings at transonic airspeeds. The authors cited 
Schewe, Günter, and Mai, Holger. "Experiments on transonic limit-cycle-flutter of a flexible swept wing." Journal of Fluids and Structures 84 (2019): pp. 153-170. http://doi.org/10.1016/j.jfluidstructs.2018.07.005

in the following probably observed some kind of LCOs, but they did not use the term. For example Moses and Pierce (1977) report about structural response of elastic wind-tunnel models, which they called "Torsional wing buzz". It may occur at high subsonic speeds as the stall develops. Then the primary torsional mode is excited by unsteady aerodynamic forces. Eckstrom et al. (1994) performed an investigation using an elastic, supercritical wing called ARW-2, which exhibits a region of high-dynamic response in the transonic speed range. Near the first-bending mode at Mach numbers around 0.92 the response of a wing-tip accelerometer exhibits amplitude-limited, nearly harmonic signals. Flutter boundaries and LCO behavior were investigated by Edwards et al. (2001) at a half-wing model MAVRIC. The influence of the dynamic pressure and of the angle of attack on the LCO behavior was reported. Maps are presented of the results of the wing-tip accelerometers depending on the Mach number and dynamic pressure and in addition unsteady pressure distributions were taken at three sections. The observed LCO behavior seems to be associated to intermittent flow separation occurring over the areas of the wing where the model exhibits dominant modal motions.

The transonic flutter experiments of Schewe et al. (1996, 2003) and Dietz et al.(2003) were focused on the investigation of nonlinear effects in as pure a form as possible. Therefore a two-dimensional rigid wing was elastically suspended at both sides in such a way that the wing had a heaving and torsional degree of freedom. Several manifestations of types of transitions and LCOs are described including the possibility to suppress or excite the flutter oscillations.

The experiments which are the base of this paper were performed in the framework of the research project "Aerostabil" and the features and details of the wing models and their structural properties are already described by Dietz et al. (2003) and in Schewe \& Mai (2018). The main aim was to study the steady aeroelastic effects (reported in Schewe \& Mai (2018), and in particular the LCO-flutter behavior of generic elastic swept wing. Thus a geometry of low complexity was selected. Nevertheless the aerodynamic shape is close to the geometry of the outer part of a modern transport aircraft wing. Based on the data published in Dietz et al. (2003), Bendiksen studied numerically LCO-flutter behavior using a very similar, theoretical model, which he called the "G-wing". In Bendiksen $(2008,2009)$ several investigations of this Aerostabil-similar wing with an identical planform but different airfoil shape and an Euler CFD-solver are presented. The amplitude limitation is explained with the "structural washout" effect, which is demonstrated to be especially important under transonic flow conditions. It was shown by Bendiksen that the load-decreasing pitch motion supports the transition from continuous to intermittent shock motion (Tijdeman \& Seebass, 1980), which decreases the aerodynamic work performed on the structure.

Schewe (2013) used the Aerostabil-experiment as one of three cases to show that the behavior of different aeroelastic systems, which are prone to flutter can partly be modelled by simple nonlinear equations. For example it is demonstrated that beyond a critical point the build-up of the oscillations and the transition to the limit-cycle, i.e. the envelope of the measured time functions, agrees with the solutions of the Landau equation. The second case was also about transonic flutter but again the two-dimensional airfoil experiment was examined, already presented in Schewe et al. (2003). The third case was about self-excited torsional oscillations of a Tacoma section i.e. a bluff body-example occurring in incompressible flow. Referring to the Aerostabil experiment, part of it was presented in Schewe (2013). In that paper the examination is focused on one special case in the transonic regime, at which the amplitudes of the limit cycle oscillations were maximal. In this context it is interesting to remark that a similar case taken at nearly the same flow conditions was selected by Stickan et al. (2014) as test case for their numerical simulation. A Navier Stokes solver (DLR-Tau code) and a linear structural shell model were applied. It turned out that the application of a shell-FE-model is 
necessary for the correct simulation of the Aerostabil experiments. Applying a dynamical single-degree-of-freedom model, derived from this structural model, a detailed computational insight into the measured LCOs is possible. The analysis shows that the nonlinear shock dynamics at the outer wing plays a dominant role for the occurrence of LCOs.

The present investigation is concentrated on the evaluation of the time functions of global forces, pressure distributions in three sections and accelerations. Flutter experiments were analysed systematically in the transonic Mach number range $0.84<\mathrm{Ma}<0.89$ and for six angles of incidence $\alpha\left(1.46^{\circ} \leq \alpha \leq 2.7^{\circ}\right)$. Maps of stability and LCO amplitudes are presented. Finally a LCO test case, already treated in the literature by the mentioned two authors is examined in more detail. This test case, containing the stage of built-up and the transition to the limit cycle provides an excellent opportunity for code validation purposes.

\section{Test set-up}

As mentioned in the introduction, the features and details of the elastic wing model and their structural properties are described in Dietz et al. (2003) and Schewe \& Mai (2018), thus here we repeat only the most important points. The sketch of the test-setup in Figure $\mathbf{2}$ shows that the swept wing model is mounted on a turntable device. The half-wing model was equipped with pressure transducers in three wing sections. In addition accelerometers were installed for getting information about the oscillating wing deflections.

The model can be forced by means of a hydraulic rotation actuator to perform pitch oscillations around the spar axis. Laser triangulators are used to measure the angle of incidence $\alpha$ at the root, related to the spar axis. A rigid piezoelectric platform balance is applied to measure the steady and unsteady global forces at the root of the wing (Schewe, 2007). The laminar-turbulent boundary-layer transition has been tripped during all the tests at $7.5 \%$ local chord length at the upper and lower surface. The experiments were performed in the adaptive test section of the transonic wind tunnel in Göttingen.

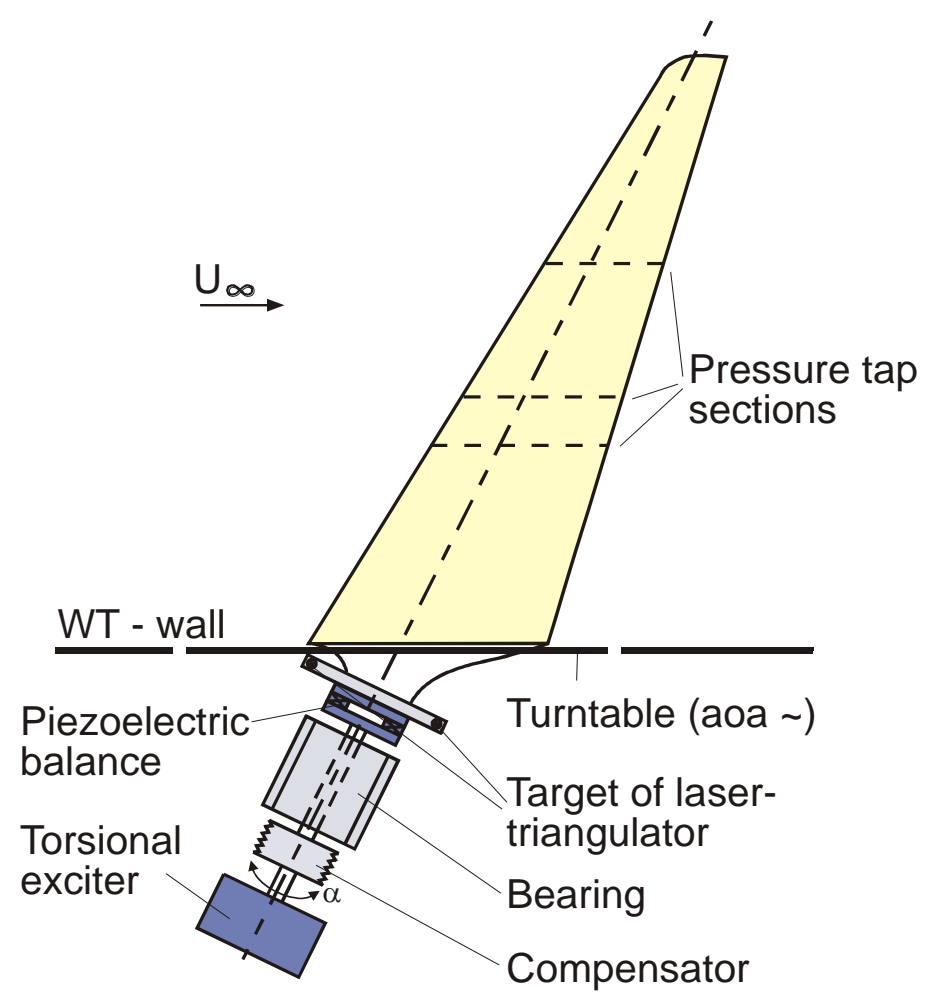


Schewe, Günter, and Mai, Holger. "Experiments on transonic limit-cycle-flutter of a flexible swept wing." Journal of Fluids and Structures 84 (2019): pp. 153-170. http://doi.org/10.1016/j.jfluidstructs.2018.07.005

Figure 2. Test setup for oscillating half models in the transonic wind tunnel. 
Schewe, Günter, and Mai, Holger. "Experiments on transonic limit-cycle-flutter of a flexible swept wing." Journal of Fluids and Structures 84 (2019): pp. 153-170. http://doi.org/10.1016/j.jfluidstructs.2018.07.005

The details of the geometry of the wing model are already sketched in Schewe \& Mai (2018). The leading-edge sweepback angle is $32^{\circ}$. The reference chord length of $\mathrm{c}^{*}=183 \mathrm{~mm}$ and a span without wing tip of $b^{*}=600.9 \mathrm{~mm}$ is used for normalization.. The wing has a supercritical airfoil and the thickness according to the local chord length remains almost constant along the span with a value of about $10 \%$.

Five accelerometers, which are regarded in this study are located in three sections. The signals were scanned with a frequency of $\mathrm{f}_{\text {scan }}=1.2 \mathrm{kHz}$ and the integration time was $\mathrm{T}=16 \mathrm{~s}$. The special flutter test case, which will be presented, was also scanned with the fourfold frequency $4.8 \mathrm{kHz}$. The first bending mode of the flexible wing without wind has a value of $\mathrm{f}_{\mathrm{B}}{ }^{*}=37.81$ $\mathrm{Hz}$ and a damping of $\delta=0.52 \%$ (second mode: $\mathrm{f}_{\mathrm{B} 2} *=112.9 \mathrm{~Hz}$ and a damping of $\delta_{\mathrm{B}}=$ $0.39 \%$ ). The first torsion mode amounts to $\mathrm{f}_{\mathrm{T} 1} *=272.6 \mathrm{~Hz}$ and a damping of $\delta_{\mathrm{T}}=0.04 \%$. The total mass of the elastic wing attached to the balance was $\mathrm{m}^{*}=6.602 \mathrm{~kg}$. The ratio of structural forces to the aerodynamic loads is described by the mass ratio:

$\mu=\left(\mathrm{m}^{*} /\left(\pi / 4 \mathrm{c}^{* 2} \mathrm{~b}^{*}\right)\right) / \rho^{*}{ }_{\infty}=417.68 \mathrm{~kg} / \mathrm{m}^{3} / \rho^{*}$. The global aerodynamic forces and moments were reduced to aerodynamic coefficients as follows: The reference area is the horizontal projection area of the wing with $\mathrm{A}^{*}=0.10033 \mathrm{~m}^{2}$. The streamwise moment center is located according to the geometrical neutral point of the wing at $x=1.047$. The spanwise moment center is located at the wind-tunnel wall $\mathrm{y}=0$ while the vertical moment center is chosen to be $\mathrm{z}=0$. The reference area $\mathrm{A}^{*}$ and the reference chord length $\mathrm{c}^{*}$ are used to normalize the global pitching-moment coefficient $\mathrm{c}_{\mathrm{m}}$. The Reynolds number Re is referred to length $\mathrm{c}^{*}$. Regarding pressure distributions $\mathrm{c}$ is the local cord length of the wing.

\section{Results: Limit-Cycle-Flutter}

In Schewe \& Mai (2018) it was mentioned that during the steady measurements limit cycle oscillations of the flexible wing were observed, which occurred in a special region of flow parameters specifically at $\mathrm{Ma}=0.86$, at higher stagnation pressure $\mathrm{p}_{0}$ and for angles $\alpha>2.2^{\circ}$. To illuminate this point, in Figure 3 a zoom of the sectional lift curve $c_{1 \_3}$ for the outer section 3 of the flexible wing is displayed, taken at two stagnation pressures $\mathrm{p}_{0}$ corresponding to a Reynolds numbers of $\operatorname{Re}=1 . e 6$ (crosses) and a higher one at $\operatorname{Re}=1.3 \mathrm{e} 6$ (circles) - the Mach number was $\mathrm{Ma}=0.86$. As mentioned at the higher pressure (i.e. higher $\mathrm{Re}$ ) there was the onset of limit cycle oscillations for angles $\alpha \geq 2.2^{\circ}$. Thus at this higher pressure the steady measurements as function of the angle of incidence had to be stopped at $\alpha=2^{\circ}$. These flutter phenomena were not expected as the theoretical calculations of the wing designer, based on the Doublet-Lattice Method had predicted that for the planned range of flow parameters the flexible wing should be free of unstable regions.

Referring to Figure 3, a detail of the lift curve for the outer pressure section was selected as this sectional lift represents the processes at the outer wing more adequately than the global lift. In addition nonlinearities even in the relevant steady values can provide indications regarding flutter oscillations. It is clear that we cannot take reasonable steady measurements in the unstable regions but even these curves we have taken at lower pressure can give an impression on how the shape could look like in the unstable regions. Indeed both lift-curves are not as straight as in case of linear behavior, there are small kinks and S-forms. As mentioned and indicated in the figure by the dotted vertical line for angles beyond $\alpha \geq 2.2^{\circ}$ and $\mathrm{p}_{0}=52 \mathrm{kPa}$ (i.e. higher Re) there was an onset of limit cycle oscillations. Consequently flutter experiments were performed systematically in the transonic Mach number range $0.84<$ $\mathrm{Ma}<0.89$ and for six angles of incidence $\alpha\left(1.46^{\circ} \leq \alpha_{0} \leq 2.7^{\circ}\right)$. 


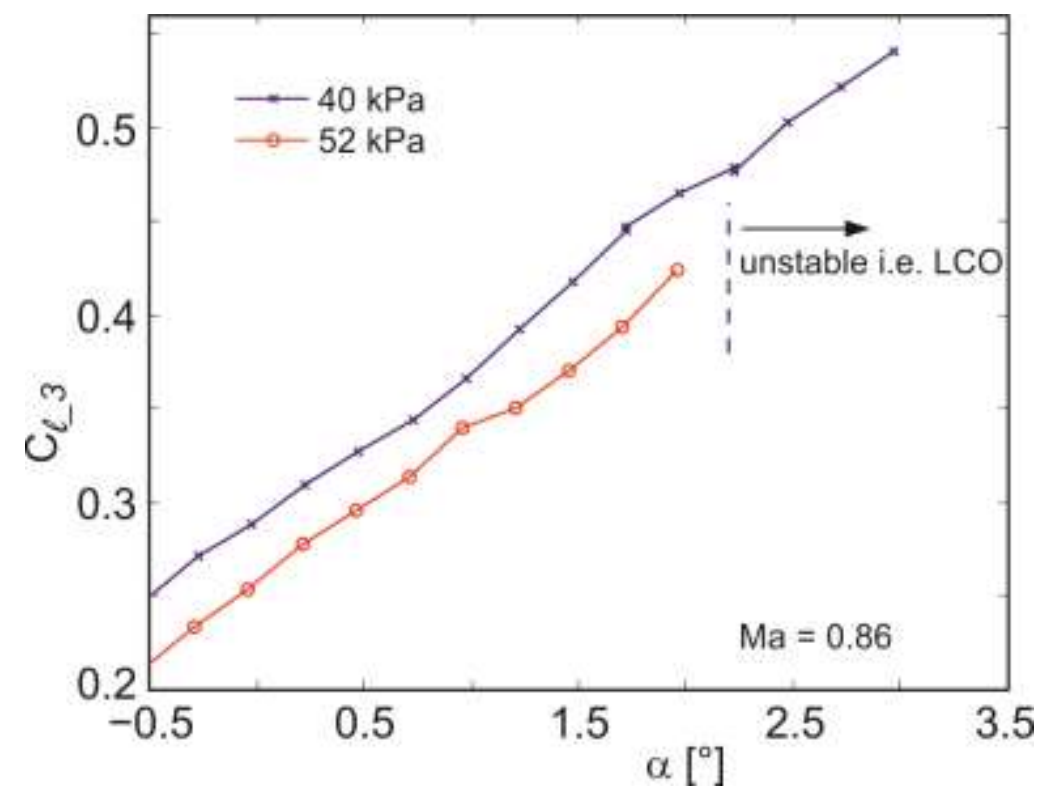

Figure 3. Zoom of the mean lift curve $c_{1 \_3}(\alpha)$ for the outer pressure section, taken at two stagnation pressures in the wind tunnel and constant Mach number Ma $=0.86$.

\subsection{Typical LCO flutter case (test case)}

A typical procedure in the beginning phase of the flutter experiments was as follows: At constant Mach number and constant angle of incidence $\alpha$ at the root of the wing, the stagnation pressure $\mathrm{p}_{0}$ in the wind tunnel was increased up to a value a little bit below the expected or assumed critical point for the onset of the oscillations. Then the angle of incidence was increased in small steps $\Delta \alpha \approx 0.1^{\circ}$ by the hydraulic actuator i.e. by a rotation around the spar axis. In Figure 4 is shown the temporal development from the onset up to the limit cycle oscillation. We see the time function of the sectional lift $\mathrm{c}_{1 \_} 3(\mathrm{t})$ i.e. the integrated pressure distribution of the outer section, displayed together with the associated time history of $\alpha$ at the root of the wing.
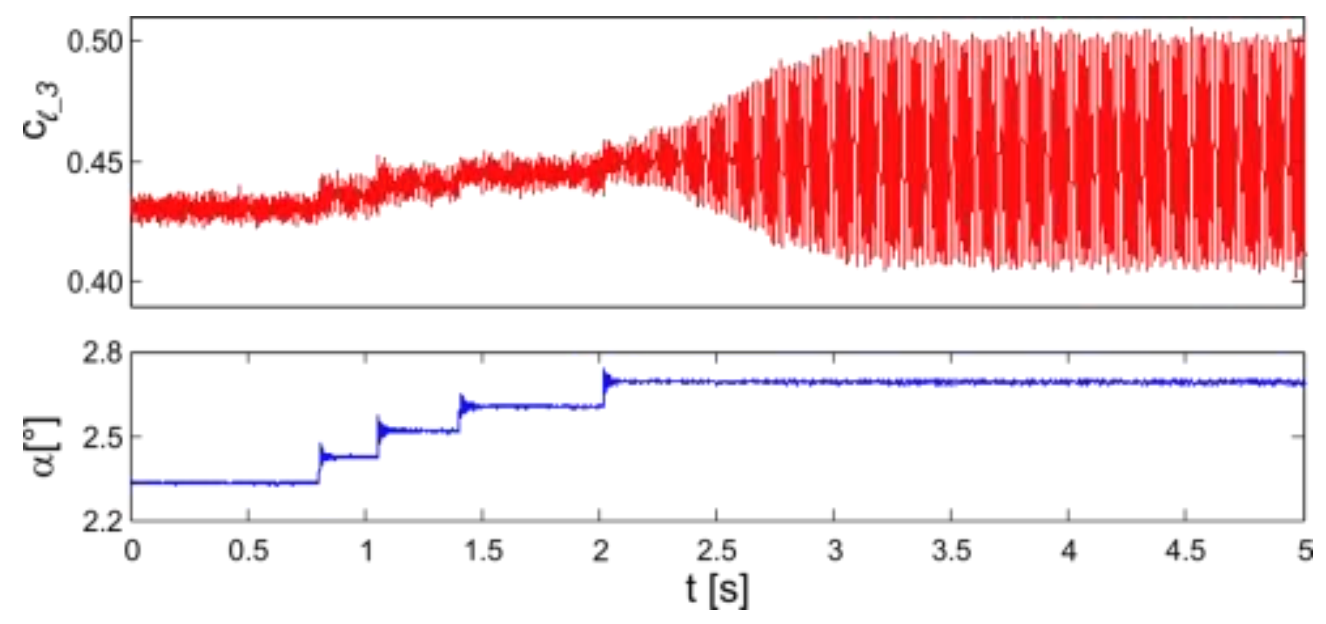

Figure 4. Time history of the sectional lift $\mathrm{c}_{1_{\_} 3}(\mathrm{t})$ around the flutter boundary (upper figure). The onset of the self-excited oscillations was initiated by a small increment of the angle of incidence $\alpha(t)$ at the root of the wing (lower figure). 
At time $\mathrm{t}=2.1 \mathrm{~s}$, beyond the last step from $\alpha=2.6^{\circ}$ to $2.7^{\circ}$ there is the onset of the selfexcited oscillation i.e. the crossover through the boundary of stability has occurred in this range of $\Delta \alpha=0.1^{\circ}$. Before the last step in $\alpha$ the small amplitude vibrations in the bending frequency are due to the excitation of the turbulent fluctuations in the oncoming transonic flow. After the final step of angle $\alpha$, self-excited oscillations set in exponentially and then there is a transition, a decay in an oscillation with constant amplitude i.e. a limit cycle oscillation. In the state of limit cycle the peak-to-peak value of the sectional lift amounts to $\Delta \mathrm{c}_{1_{-} 3} \approx 0.1$ and as the measurement is based on integrated pressures, there is no contribution of inertia effects.

This measuring point can be seen as a paradigmatic one and is labelled as " $\mathrm{R} 1520 \mathrm{MP}$ 5". It is a special one, because the oscillation amplitudes were the highest in our experiments. In addition and also important and obvious in Figure 4, we have captured the whole time history of the flutter phenomenon including the transition from stable/steady to unstable/unsteady. The sampling frequency $(4.8 \mathrm{kHz})$ was fourfold compared to the usual value.

Using the balance and the accelerometer signals the frequency during LCO was determined to $\mathrm{f}_{\mathrm{f}} *=50.24 \mathrm{~Hz}$. Then the relevant characteristic values are as follows: The measurement was taken at $\mathrm{Ma}=0.865$, a stagnation pressure of $\mathrm{p}=70 \mathrm{kPar}$ and a Reynolds number $\mathrm{Re}=1.68$. $10^{6}$, the flutter-index amounts to $\mathrm{Fi}=2 \mathrm{u}_{\mathrm{red}} / \sqrt{ } \mu=0.49$ with $\mathrm{u}_{\mathrm{red}}=\mathrm{u}_{\infty} /\left(2 \pi \mathrm{f}_{\mathrm{B} 1} \mathrm{c}^{*}\right)$ and the reduced frequency is $\mathrm{k}=2 \pi \mathrm{f}_{\mathrm{f}} \mathrm{c} * / \mathrm{u}_{\infty}=0.202$. The limit cycle amplitude at the position of the outer accelerometer is $h_{\text {peak }}=\sqrt{ } 2 h_{\text {RMS }}=0.009 \mathrm{~m}$. Performing an extrapolation based on the accelerometer signal a rough estimate of the wing tip deflection yields an amplitude of $0.0126 \mathrm{~m}$.

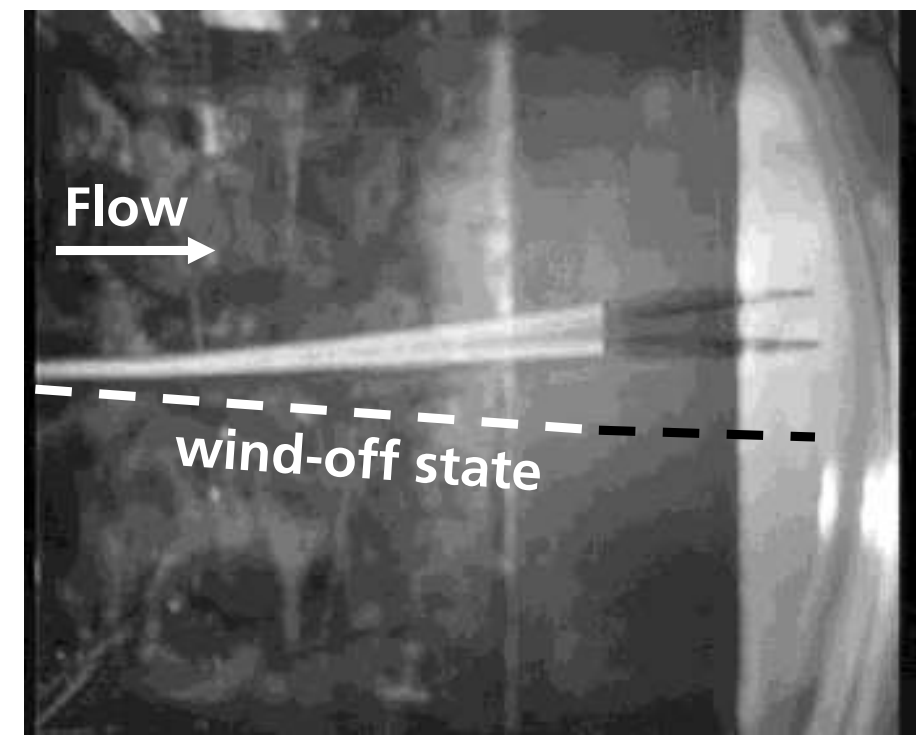

Figure 5. Snapshot of the half wing, twisted and bent by airloads during limit-cycle-flutter (Figure from Dietz et al. 2003)

In Figure 5 there is a typical snapshot taken during the measurement described before. The picture was extracted from a movie and shows the outer part of the wing, which is twisted and bent by the steady air loads. In addition, the fact that the wing is undergoing flutter i.e. in the 
state of a limit-cycle-oscillation is very well documented. Because the movie was taken by an observation camera, whose exact position is not yet known, only a rough estimate is possible concerning the steady and unsteady deformation. The heaving amplitude is then about $0.013 \mathrm{~m}$ at the leading edge. This value is not too far away from the accelerometer measurements regarding the uncertainties and assumption of the unknown position of the camera. Considering in particular the wing tip, it is obvious that the heaving is superimposed over a rotational motion. The combined motion is like a rotation around an axis ahead the leading edge i.e. a single degree of freedom oscillation.

\subsection{Maps of stability}

Apart from these singular points flutter experiments were performed systematically in the Mach number range $0.84<\mathrm{Ma}<0.89$ in steps of $\Delta \mathrm{Ma}=0.005$ and for six angles of incidence $\alpha$ at the root of the wing $\left(1.46^{\circ} \leq \alpha \leq 2.7^{\circ}\right)$. The procedure of the flutter experiments was as follows: At constant Mach number and constant angle of incidence $\alpha$ at the root of the wing, the stagnation pressure $\mathrm{p}_{0}$ in the wind tunnel was increased from about $40 \mathrm{up}$ to $70 \mathrm{kPa}$ and the measurements were taken in steps of $\Delta \mathrm{p}_{0}=2$ to $4 \mathrm{kPa}$. Such a pressure sweep was applied as long as the amplitudes remained in reasonable limits as in the following case.

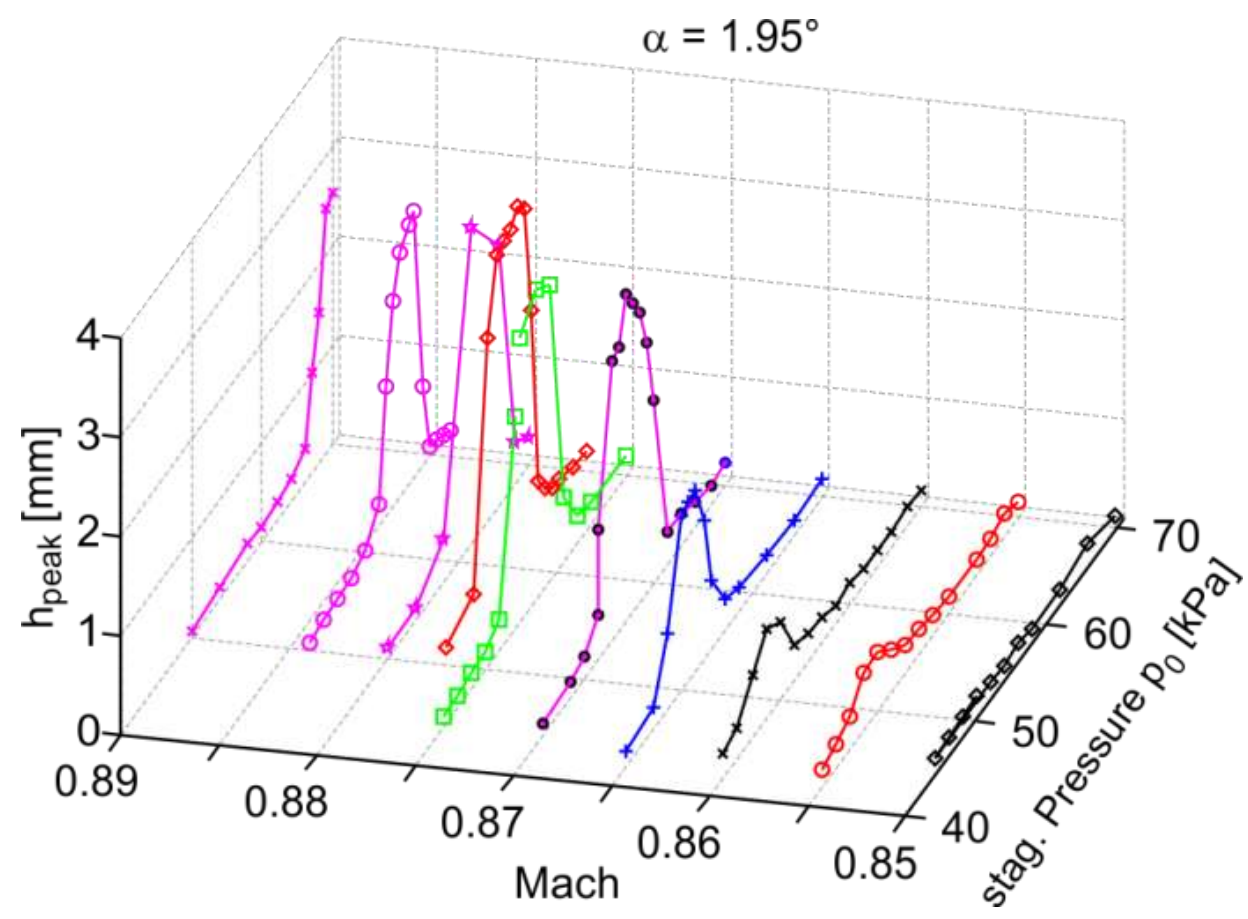

Figure 6. Limit cycle amplitude $\mathrm{h}_{\text {peak }}$ as function of the stagnation pressure $\mathrm{p}_{\mathrm{o}}$ in the wind tunnel and the Mach number, measured at $79 \%$ of wing-span.

The angles of incidence is $\alpha=1.95^{\circ}$.

Figure 6 represents a typical measurement, showing the limit cycle amplitude $\mathrm{h}_{\text {peak }}$ as function of the stagnation pressure $\mathrm{p}_{\mathrm{o}}$ and the Mach number. The angle of incidence was held constant at $\alpha=1.95^{\circ}$. The amplitude $\mathrm{h}_{\text {peak }}$ was obtained by twofold integration of the mean of the two accelerator-signals at $y / b^{*}=0.79$ (b*: wing span without wing-tip). The main feature of the curves is the observation that with increasing stagnation pressure the vibration 
amplitude $\mathrm{h}$ is not monotonically increasing, on the contrary at medium high pressure there is a reversal of $h_{\text {peak }}\left(p_{0}\right)$ i.e. the amplitude decreases with increasing $p_{0}$ and finally at even higher values of $\mathrm{p}_{0}$ the vibrations cease. With other words: unstable regions are islands, which can be approached from both sides i.e. also for decreasing stagnation pressure $\mathrm{p}_{0}$. This seems to be a special property of an elastic swept wing, as the local angle of attack changes depending on the stagnation pressure. This aeroelastic effect can have consequences on the flutter behavior. Considering Figure 6 we can state:

(i) The range of instability begins between $\mathrm{Ma}=0.85-0.855$.

(ii) With increasing Mach the onset of the LCOs is shifted to higher pressure $\mathrm{p}_{0}$ (contrary to transonic dip).

(iii) The curves are looking like resonance curves, which are a little bit bent to higher stagnation pressure, a fortiori the Mach increases. In the end the bending of the curves seems to lead to hysteresis ranges, particularly at the side of higher Mach numbers.

(iiii) With increasing Mach the amplitude $\mathrm{h}_{\text {peak }}$ increases up to maximal values of $4 \mathrm{~mm}\left(\mathrm{~h}_{\text {peak }}\right.$ $/ \mathrm{c}^{*}=0.022$ ) at about $\mathrm{Ma}=0.875$, then there is a slight decrease.

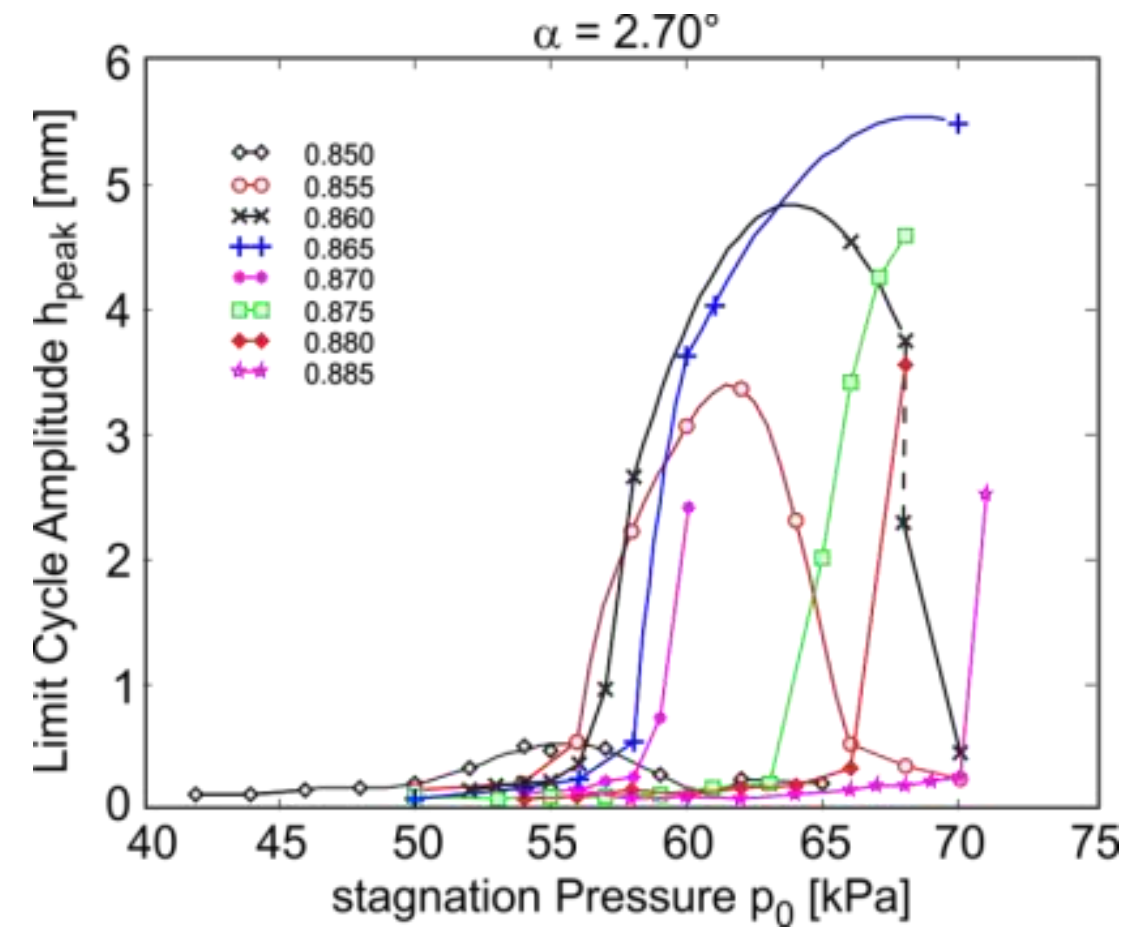

Figure 7. Limit cycle amplitude depending on the pressure $\mathrm{p}_{\mathrm{o}}$ in the wind tunnel and the Mach number as parameter. The angle of incidence is $\alpha_{0}=2.7^{\circ}$.

In Figure 7 a corresponding measurement taken at a higher angle of incidence $\alpha=2.7^{\circ}$ is displayed. It shows also the limit cycle amplitude depending on the pressure $\mathrm{p}_{\mathrm{o}}$, but in this plane representation the Mach number is only a parameter thus the individual curves are not as clearly separated, nevertheless a quantitative evaluation and assessment of the curves is easier and more accurate. The increased angle of incidence has the following consequences: (i) Because of the high amplitudes $h$, in particular at higher Mach, the range of instability could not be crossed by continuously increasing the pressure $\mathrm{p}_{0}$ as it could have been done at the lower angle $\alpha_{0}=1.95^{\circ}$. Considering for example the case of $\mathrm{Ma}=0.86$ (symbol $\mathrm{x}$ ), the unstable regime had to be approached from two sides i.e starting with low pressure $\mathrm{p}_{0}$ and high pressure, respectively. 
Schewe, Günter, and Mai, Holger. "Experiments on transonic limit-cycle-flutter of a flexible swept wing." Journal of Fluids and Structures 84 (2019): pp. 153-170. http://doi.org/10.1016/j.jfluidstructs.2018.07.005

(ii) The range of instability begins a little bit below $\mathrm{Ma}=0.85$.

(iii) Also here with increasing Mach the onset of the LCOs is shifted to higher pressure.

(iv) The curves are a little bit curved to higher pressure, a fortiori the Mach increases. At Ma $=0.86$ there is probably a hysteresis range (dotted).

(v) With increasing Mach the amplitude $h_{\text {peak }}$ increases steeper than a lower $\alpha$.

For Ma $>0.865$ after the onset of LCO the increase of the amplitudes $h_{\text {peak }}\left(\mathrm{p}_{0}\right)$ is so steep that as mentioned the pressure was not increased further. Thus the measuring point taken at $\mathrm{p}_{0}=$ $70 \mathrm{kPa}$ and $\mathrm{Ma}=0.865$ (symbol + ), corresponding to the already discussed MP "R $1520 \mathrm{MP}$ 5" was approached applying a different procedure: As described in detail in the beginning of the chapter, the state $(70 \mathrm{kPa}, \mathrm{Ma}=0.865)$ was approached at reduced angle $\alpha \approx 2.3^{\circ}$ (lower Figure 4). Then the desired state was reached by stepwise increase up to $\alpha=2.7^{\circ}$.

Referring to Figure 7 it is possible to obtain limits of stability for every Mach number if one looks for the locations, where the curves $h_{\text {peak }}\left(p_{0}\right)$ cross a special threshold value of $h_{\text {peak }}$. The selection of a threshold value seems to be a little bit arbitrary, but we have no other choice. We select $h_{\text {peak }}=0.5 \mathrm{~mm}$, a value which is slightly higher than the vibration amplitude anyway excited by the turbulent fluctuations of the oncoming transonic flow. The line connecting the intersection points leads to the outer contour-line in the diagram of stability $\mathrm{p}_{0}(\mathrm{Ma}, \alpha=$ const) as plotted in Figure 8. The small red points correspond to the individual measurement points (MPs), which can also be found in Figure 7. In addition the contour lines for higher limit cycle amplitude $h_{\text {peak }}=2,3.5$ and 5 are included. Again the outer line for $h_{\text {peak }}=0.5 \mathrm{~mm}$ shall be seen as the boundary of stability. Considering the contour lines (Figure 8) some features we have extracted from Figures 6,7 are now more obvious, in particular the fact that at high values of the pressure $\mathrm{p}_{0}$ the vibrations cease again. Therefore the shape of the unstable area looks like an island. At the first glance we see that with increasing Mach number the onset of the LCOs is shifted to higher pressure $\mathrm{p}_{0}$. The range of instability begins a little bit below Ma $=0.85$. With increasing Mach the limit cycle amplitude $\mathrm{h}_{\text {peak }}$ increases and can exceed values of $5 \mathrm{~mm}\left(\mathrm{~h}_{\text {peak }} / \mathrm{c}^{*}=0.027\right)$ at about $\mathrm{Ma}=0.875$. In this representation the already discussed flutter point MP "R $1520 \mathrm{MP} 5$ " can be found at $\mathrm{p}_{0}=70 \mathrm{kPa}$ and $\mathrm{Ma}=0.865$.

As mentioned at the begin of the chapter such measurements were taken for 6 angles of incidence $\alpha=1.46^{\circ}, 1.71^{\circ}, 1.95^{\circ}, 2.21^{\circ}, 2.45^{\circ}$ and $2.7^{\circ}$. The response curves for $\alpha=1.95^{\circ}$ and $2.7^{\circ}$ were already presented, the first example was selected as the oscillation amplitudes for the entire pressure sweep remained in reasonable limits therefore we have got a complete curve $h_{\text {peak }}\left(\mathrm{p}_{0}\right)$ in one pressure run i.e. without interruption. The second case $\alpha=2.7^{\circ}$ (Figure 7 ) is an example where the oscillation amplitudes in the middle of the unstable region are too high, consequently we had to approach the unstable area from both sides. Anyhow we have concentrated on this case, in particular on the measuring point MP " $R 1520$ MP 5" because for this test case the amplitudes are highest and thus the effects and phenomena are most pronounced.

In order to obtain the diagrams of stability belonging to the so far missing angles of incidence we used the same procedure as described. Hence also the boundaries of stability in the plane of Mach and tunnel pressure were determined for $\alpha=1.46^{\circ}, 1.71^{\circ}, 2.21^{\circ}$ and $2.45^{\circ}$. The resulting contour lines for all six angles $\alpha$ are plotted in the 3 dimensional representations shown in Figure 9. For example the third contour line from the bottom corresponds to state $\left(\alpha=1.95^{\circ}\right)$ displayed in Figure 6. The Mach number, the stagnation pressure and the angle of incidence span a three-dimensional space, in which the contour lines represent cross-sections of a three-dimensional body, inside the body the state of the aeroelastic system is unstable outside stable. 


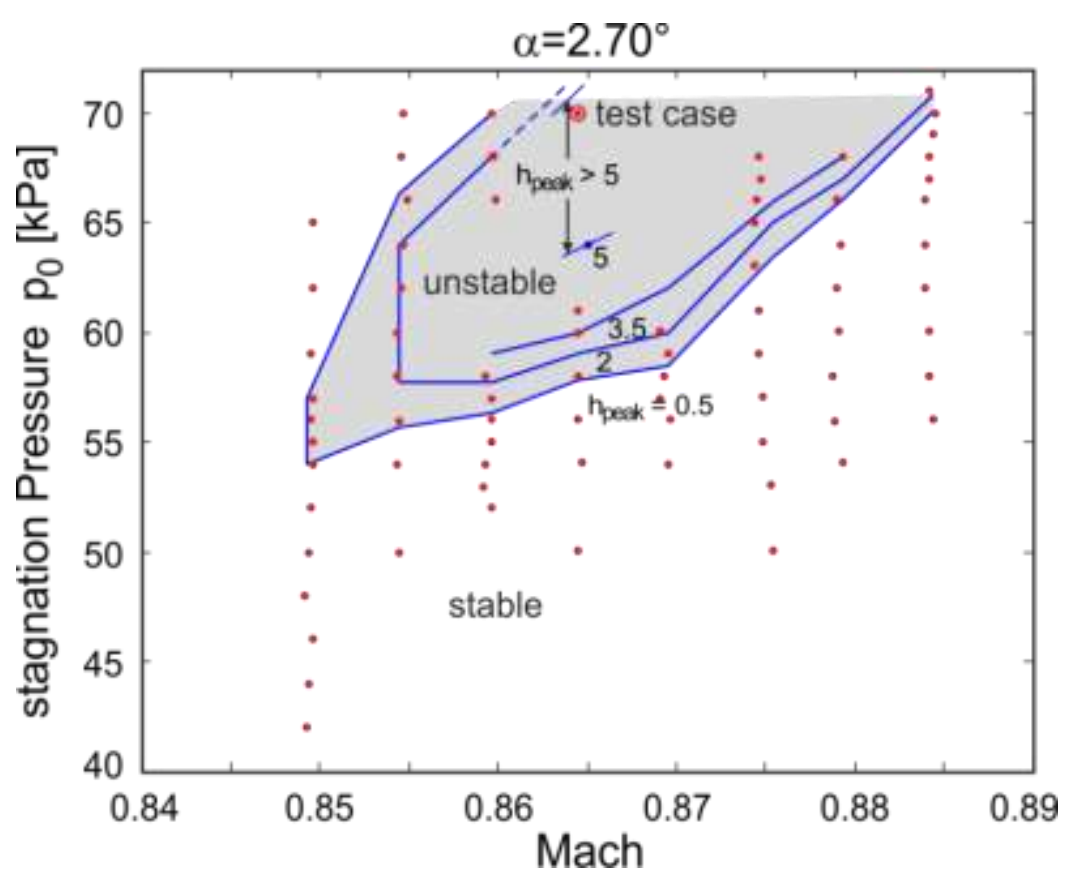

Figure 8. Diagram of stability based on the data in the preceeding figure. The small red points correspond to the individual measuring point (MPs), which were taken.

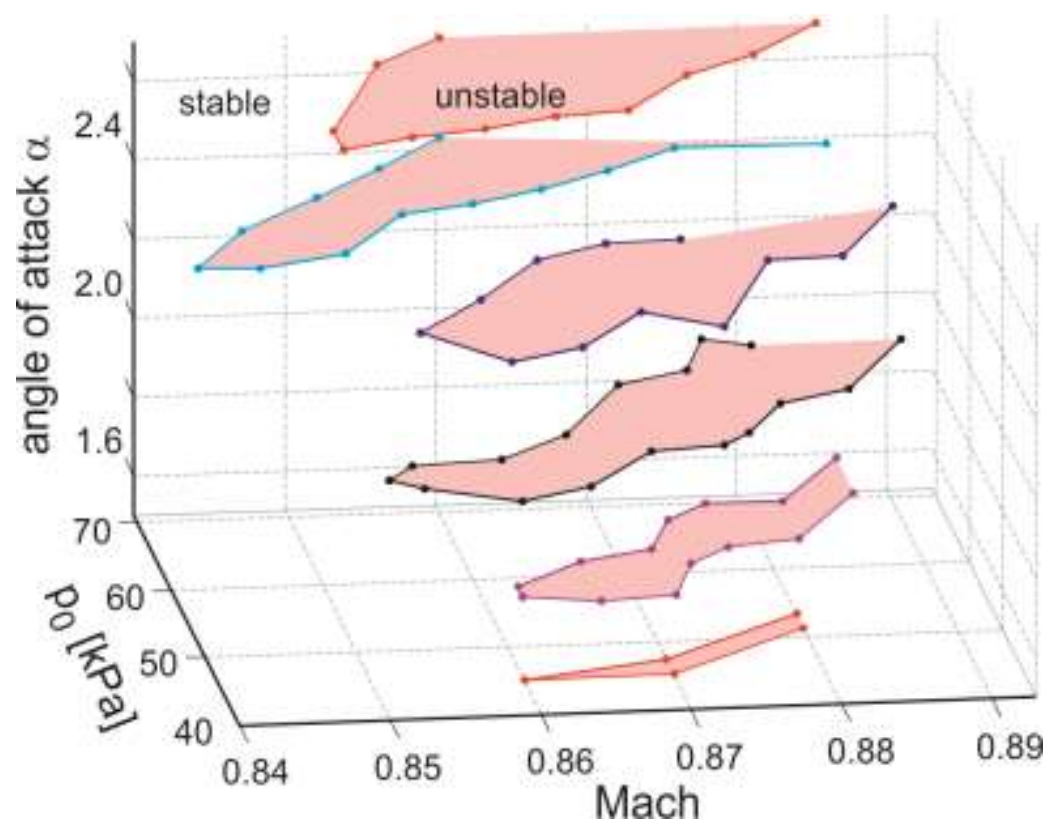

Figure 9. Boundaries of stability in the plane of Mach number and tunnel pressure.

Measurements were taken for 6 angles of incidence $\alpha$ at the root of the wing. The values are: at the bottom plane $\alpha=1.46^{\circ}$, then $1.71^{\circ}, 1.95^{\circ}, 2.21^{\circ}, 2.45^{\circ}$ up to $2.7^{\circ}$.

One could be tempted to smooth the obvious scattering of the individual contour lines but regarding notches or kinks in the curves it is difficult to distinguish between measurement scatter and real physical effects. The complicated interaction between the shocks and the 
separation in the transonic regime can lead to significant changes in the global behavior and thus to a complicated shape of boundaries of stability. Considering the upper red line in the top, we recognize that it corresponds to the outer contour line in the diagram of stability in Figure 8. This area or island seems to have the largest width. At angle $\alpha=2.45^{\circ}$ the width is considerable smaller but comparing all six cases the flutter-onset Mach number is lowest here at about $\mathrm{Ma}=0.84$. Examining the following cases from $2.21^{\circ}$ down to $1.46^{\circ}$, we can state that the islands become smaller and that a little bit below $1.46^{\circ}$ the area of instability probably would have disappeared.

\subsection{Limit Cycle Flutter after decreasing air density}

In the context of the response curves $\mathrm{h}_{\text {peak }}(\mathrm{Ma}), \mathrm{h}_{\text {peak }}\left(\mathrm{p}_{0}\right)$ and diagrams of stability (Figures 69) it was mentioned that the unstable regions are islands, such that an unstable region can be approached from both sides i.e. also for decreasing tunnel pressure $\mathrm{p}_{0}$. This seems to be a special property of an elastic swept wing, which can lead to a re-twist of the wing for different loading conditions. This is an aeroelastic effect, which can have drastic consequences on the flutter behavior. Already Bendiksen (2009) pointed out that a re-twisted wing is more prone to flutter and called the effect "High Altitude Flutter".

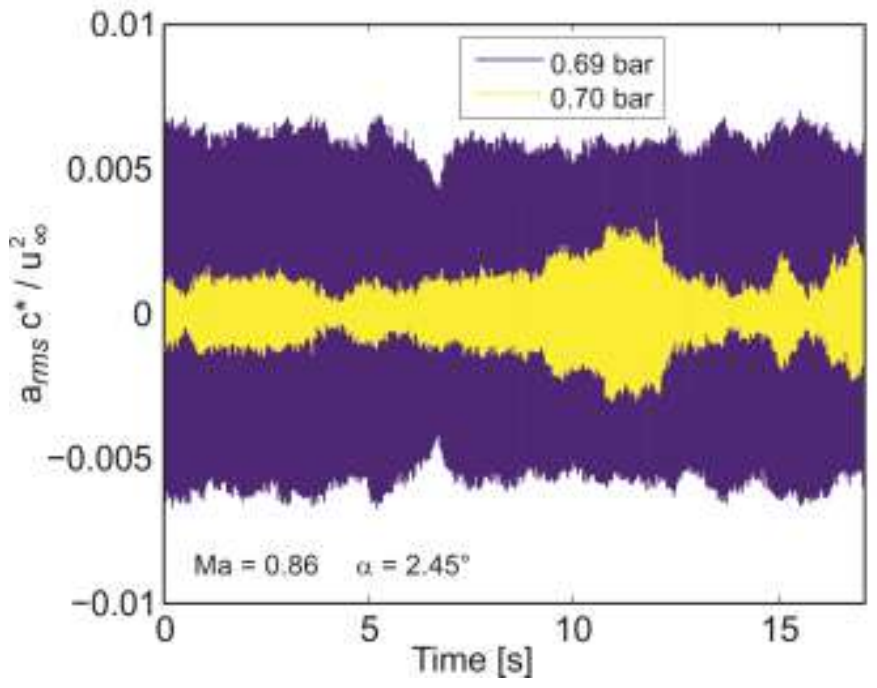

Figure 10. Two accelerometer signals, taken at different stagnation pressure $\mathrm{p}_{0}$ near the boundary of stability. The onset of LCO was effected by reducing the pressure from $\mathrm{p}_{0}=70$ to $69 \mathrm{kPa}$ i.e. a reduction of $\Delta \mathrm{p}_{0} \approx-1.4 \%$.

A drastic example is visualized in Figure 10, where two time functions of the wing tip accelerometer $\mathrm{a}(\mathrm{t})$ are plotted for two critical states. Both measurements were taken near the boundary of stability at $\mathrm{Ma}=$ const $=0.86$ and $\alpha_{0}=$ const $=2.45^{\circ}$, but the stagnation pressure $\mathrm{p}_{0}$ was reduced a little bit. The measurement was started at $\mathrm{p}_{0}=70 \mathrm{kPa}$ and the corresponding time signal is rather intermittent and seems to have critical character, the state is obviously immediately before a transition. After the reduction of the pressure $\mathrm{p}_{0}$ from 70 to $69 \mathrm{kPa}$ there is a significant jump in the oscillation amplitude by a factor of nearly 5 . The transition was effected by a relatively small reduction of $\Delta \mathrm{p}_{0}=1 / 70 \approx 1.4 \%$. All the signs are that this transition is hysteretic. The intermittency in the time function before the transition indicates that the system is testing a new state, but the fluctuations are not large enough to reach the 
new branch or state of stability - the LCO. After the transition caused by - $\Delta \mathrm{p}_{0}$ there is a nearly sinusoidal oscillation with relatively constant amplitude i.e. a limit cycle oscillation. The explanation of Bendiksen (2009) is as follows: The reduction of the pressure leads to a structural wash-in effect i.e. less bending of the swept wing is coupled with an increase of the angle of attack of a stream wise segment of the wing. As we already know from the present results, an increase of the angle of attack has a destabilizing effect, because the islands of instability become larger and the limit cycle amplitudes higher, respectively.

Finally the statement of Bendiksen was based on numerical simulations and at that time there was no experimental evidence. We could not find an experimental confirmation of "High Altitude Flutter" in the literature.
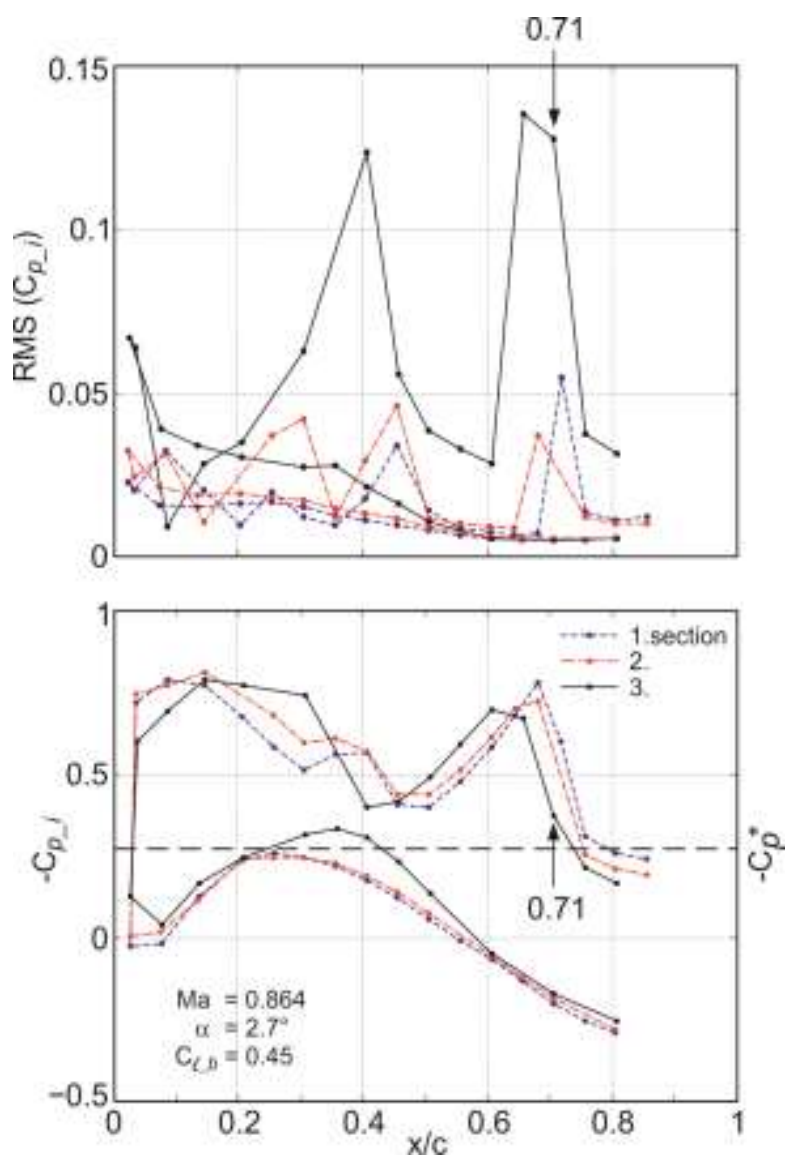

Figure 11. Mean pressure - (lower figure) and RMS-pressure distributions (upper) of the three sections $(i=1,2,3)$ during limit cycle flutter. The location indicated by arrows plays a prominent role regarding flutter behavior (see later discussion).

\subsection{Pressure distributions and deformations during limit cycle flutter}

Regarding the fluid dynamic aspects the evaluation is focused on the special measuring point "R 1520 MP 5" because for this test case the oscillation amplitudes of the wing are highest and thus the fluid dynamic effects and phenomena are most pronounced. In the lower diagram of Figure 11 the mean pressure distributions $\mathrm{c}_{\mathrm{p}_{-} \mathrm{i}}(\mathrm{x} / \mathrm{c})$ of the three pressure sections $(\mathrm{i}=1,2,3)$ are presented, which were taken during limit cycle flutter. The state of the flow is characterized by $\mathrm{Ma}=0.865$, the global lift coefficient $\mathrm{c}_{1 \_\mathrm{b}}=0.45$ and the critical pressure $\mathrm{c}_{\mathrm{p}}{ }^{*}$ $=-0.267$. The three sectional lift coefficient amount to $c_{1 \_1}=0.48$ for the inner section, $c_{1 \_2}=$ 
Schewe, Günter, and Mai, Holger. "Experiments on transonic limit-cycle-flutter of a flexible swept wing." Journal of Fluids and Structures 84 (2019): pp. 153-170. http://doi.org/10.1016/j.jfluidstructs.2018.07.005

0.48 for the middle and $c_{1 \_3}=0.42$ for the outer section. Considering the steady pressure distribution in Figure 17 in Schewe \& Mai (2018) we can conclude that the state of the flow is somewhat before the transition from double shock to single shock. In the curve it is evident that in the first pressure section the first shock is located most upstream and the second one most downstream, examining the middle (second section) and finally the outer section (third section) then the trend is in such a way that the shocks are moving toward each other. That means that compared with both other sections in the outer one the first shock is most downstream and the rear shock most upstream, in addition the steepness i.e. the shock strength of the first shock is maximal. Only on the lower side of the outer section the pressure falls below the critical value $\mathrm{c}_{\mathrm{p}} *$ between $\mathrm{x} / \mathrm{c} \approx 0.3-0.45$.

In the upper Figure 11 the corresponding RMS- pressure distributions for the three pressure sections ( $i=1,2,3)$ are displayed. In particular for the outer section (solid) the RMS at the upper side has sharp peaks at the location of the shocks with values up to 0.13 , while the RMS values at both inner sections are considerably smaller with values up to 0.05 . One reason for the different values is the fact that the oscillation amplitudes are much higher at the outer section (see later Figure 19).
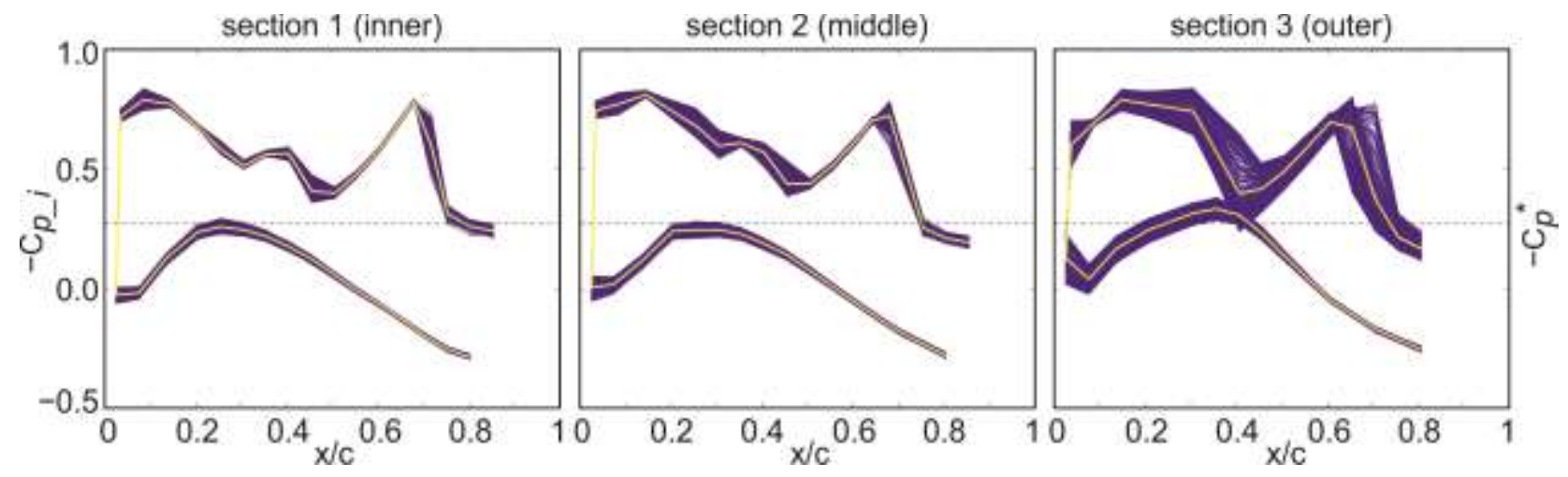

Figure 12. Superposition of instantaneous pressure distributions during Limit-CycleOscillation. The time-averaged distribution is yellow $\left(\mathrm{c}_{\mathrm{p}_{-}}, \mathrm{i}=1,2,3\right)$.

In the next Figure 12 the causes are more evident. In order to illuminate the unsteady effects a superposition of instantaneous pressure distributions is displayed taken during Limit-CycleOscillation. For comparison the time-averaged distributions from the preceding lower Figure 11 are also included as yellow lines. Again in all pressure distributions, there is a double shock on the upper side. Comparing the individual sections, the fluctuations are rather small at the inner and moderate at the middle section at the outer section there are strong fluctuations particularly around both shock locations. In the outer section the strength of the first shock is rather high, the shock is moving a large amount and the instantaneous pressure at the shock foot falls below the critical value $\mathrm{c}_{\mathrm{p}}{ }^{*}$.

In Figure 13 there are two extreme cases of instantaneous pressure distributions of the outer section, taken at time around $t=4 \mathrm{~s}$ (see Figure 4 ) and the data are extracted from Figure 12. Again for comparison the time-averaged distributions are also included with solid yellow lines. The dotted line is the instantaneous pressure distribution at minimal lift coefficient $\mathrm{c}_{1 \_}{ }_{\min }$. Here the shock positions are extremal: the first shock is most upstream and the rear shock is most downstream, in both cases the steepness i.e. shock strength is maximal. The dot/dash line is the instantaneous pressure distribution at maximal lift coefficient $\mathrm{c}_{1 \_3}{ }_{\max }$. Compared to the dotted curve corresponding to minimal lift, the shock positions here are vice 
Schewe, Günter, and Mai, Holger. "Experiments on transonic limit-cycle-flutter of a flexible swept wing." Journal of Fluids and Structures 84 (2019): pp. 153-170. http://doi.org/10.1016/j.jfluidstructs.2018.07.005

versa: the first shock is most downstream and the rear shock most upstream and the steepness (shock strength) is minimal. Finally we can conclude that firstly each shock is moving back

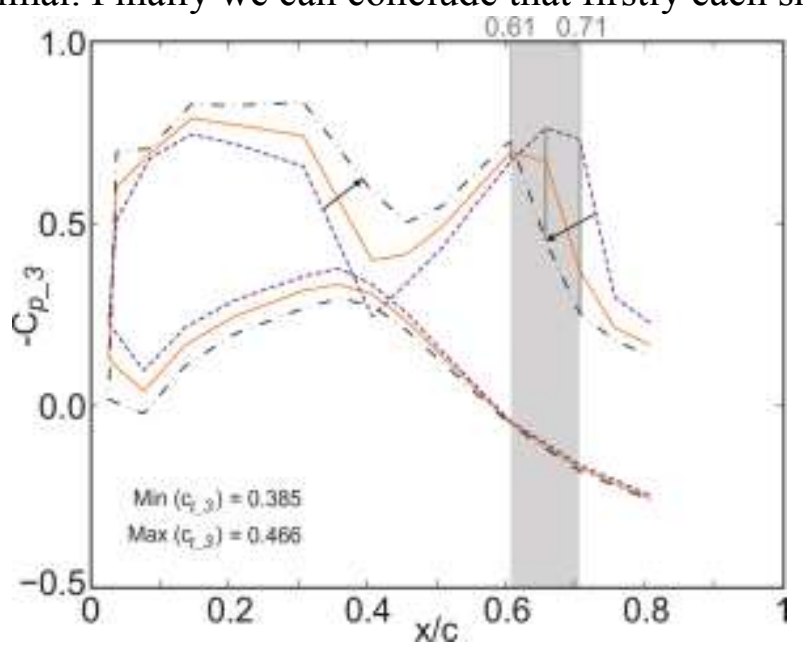

Figure 13. Both extreme cases of the instantaneous pressure distribution during LCO (outer pressure section). Time-averaged distributions are yellow. The range of the second shock motion is shaded $(0.61<\mathrm{x} / \mathrm{c}<0.71)$ and the three time functions of the pressures in this regime will be investigated in detail in a later part of the chapter (Figure 17).

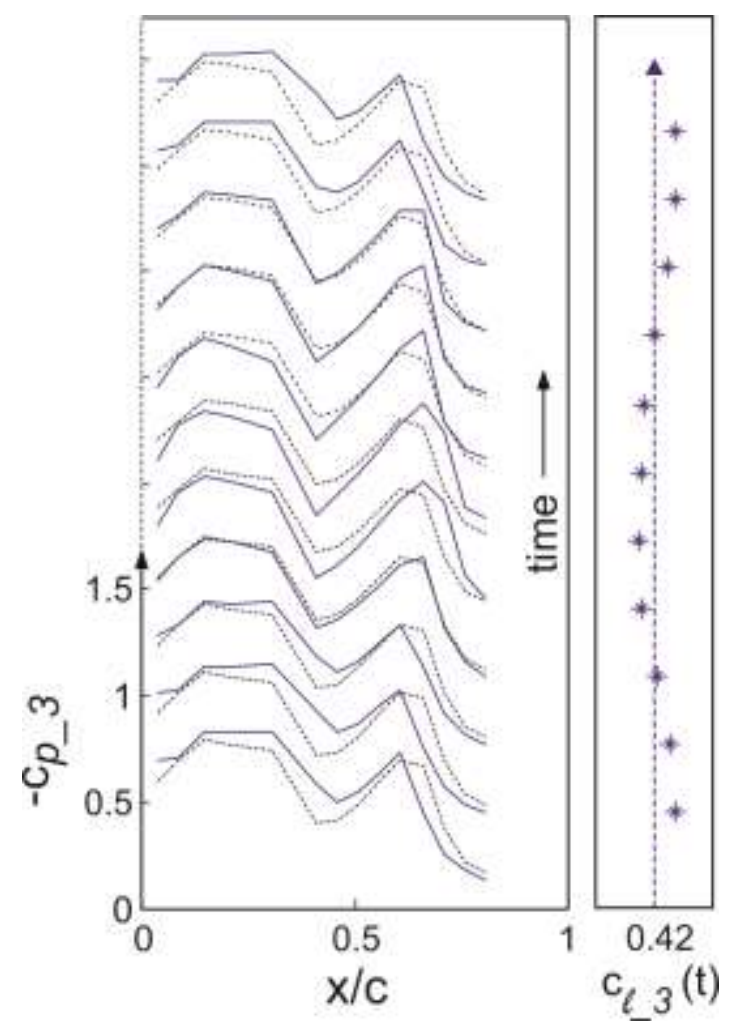

Figure 14. Instantaneous pressure distributions of the upper side (section 3) depending on time. 


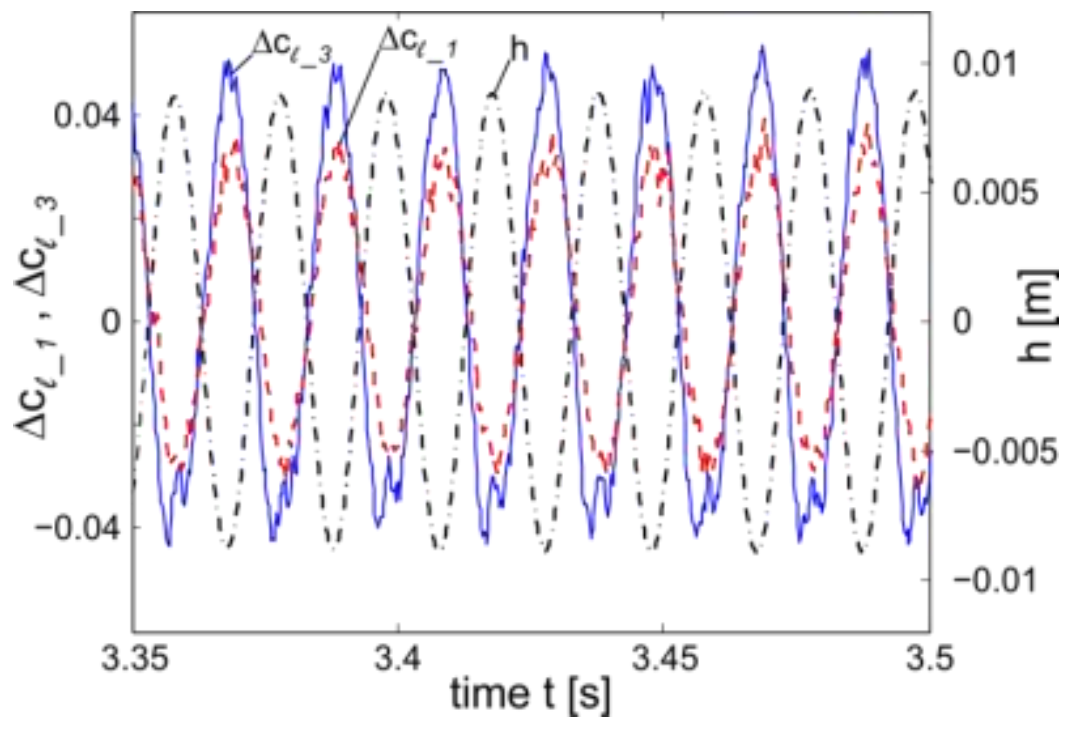

Figure 15. Time histories of displacement $\mathrm{h}$ derived from the wing tip accelerometer and the fluctuations of the sectional lifts $\Delta \mathrm{c}_{1_{1} 1}$ and $\Delta \mathrm{c}_{1_{-} 3}$ for test case "R 1520 MP 5".

and forth in the range of about $\Delta \mathrm{x} / \mathrm{c} \approx 0.1$ and secondly that in all observed cases the shocks are moving towards each other.

In order to provide a better insight in the shock dynamics for about one oscillation cycle, instantaneous pressure distributions (every tenth of the period) of the upper side against time are plotted in Figure 14. For comparison also here the time-averaged distributions are included (dashed) in the figure as well as the value of the corresponding sectional lift $\mathrm{c}_{1 \_3}$. The time is running in the direction of the ordinate and the time step is $\Delta t=2 \mathrm{~ms}$. We can observe the development of the shock strength depending on time. During downstream motion the steepness of the first shock is weakening while the steepness of the second shock is increasing and vice versa. In the next Figure 15 we see the interrelation between the lift and the motion of the wing. The figure shows the time histories of displacement $h$ derived from the wing tip accelerometer and the fluctuations of the sectional lifts $\Delta \mathrm{c}_{1 \_1}$ and $\Delta \mathrm{c}_{133}$ for the special test case "R $1520 \mathrm{MP} 5$ ". Also in the following the $\Delta$ indicates that the mean sectional lift is subtracted. It is obvious that both lift forces and the displacement are out of phase. The computation of the cross spectral density confirms the statement. That means the lift is maximal at the bottom reversal point and vice versa. Regarding the mentioned wash-in effect and from a quasi-static point of view this behavior seems to be plausible: at the bottom reversal point i.e. $h_{\min }$ the bending of the wing is minimal and consequently the angle of attack higher $+\Delta \alpha$ leading to higher lift $\Delta \mathrm{c}_{1 \_}, \Delta \mathrm{c}_{1 \_3}$ and vice versa.

Close inspection of the time histories of the sectional lifts $\Delta \mathrm{c}_{133}$ shows that in the top reversal point of displacement $\mathrm{h}(\mathrm{t})$ there is a small wobble in $\Delta \mathrm{c}_{1-3}(\mathrm{t})$ leading to an asymmetry in the negative and positive peak amplitudes. This effect can better be seen in the probability density functions $\mathrm{P}\left(\Delta \mathrm{c}_{1_{-} \mathrm{i}}\right)$ of the three sectional lifts $\Delta \mathrm{c}_{1_{-} \mathrm{i}}(\mathrm{i}=1-3)$ in Figure 16. The amplitudes $\Delta \mathrm{c}_{1_{-} \mathrm{i}}$ are referred to the corresponding RMS-values, which amount to: $\operatorname{RMS}\left(\Delta \mathrm{c}_{1 \_1}\right)=0.017$, $\operatorname{RMS}\left(\Delta \mathrm{c}_{1 \_2}\right)=0.022$ and $\operatorname{RMS}\left(\Delta \mathrm{c}_{1 \_3}\right)=0.032$. The normalisation implies that the area below each curve is one. The PDF of the inner and the middle section is nearly symmetric and similar as for a sinusoidal behavior, which is superimposed with noise. However in the outer section there is an asymmetry and the contribution of the noise is increased. The reason for the asymmetry is the small wobble, occurring in the bottom reversal point of the sectional lift 
Schewe, Günter, and Mai, Holger. "Experiments on transonic limit-cycle-flutter of a flexible swept wing." Journal of Fluids and Structures 84 (2019): pp. 153-170. http://doi.org/10.1016/j.jfluidstructs.2018.07.005
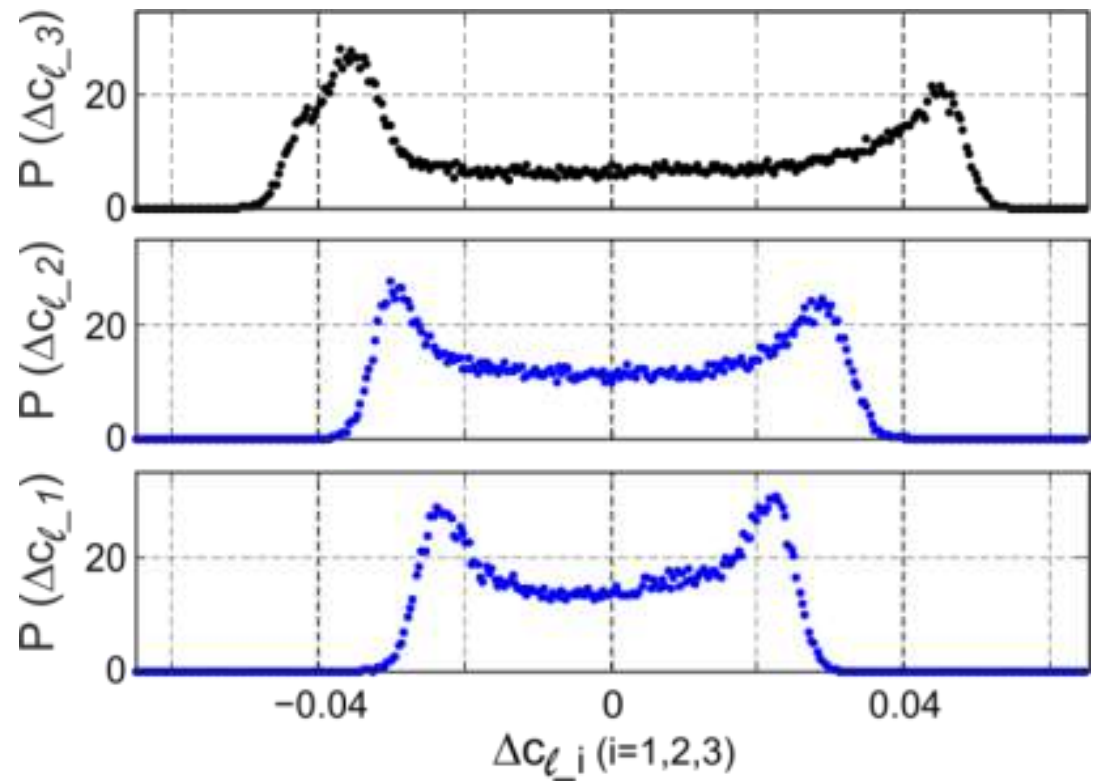

Figure 16. Probability density functions of the sectional lifts $\Delta \mathrm{c}_{1 \_i}(\mathrm{i}=1,3)$.

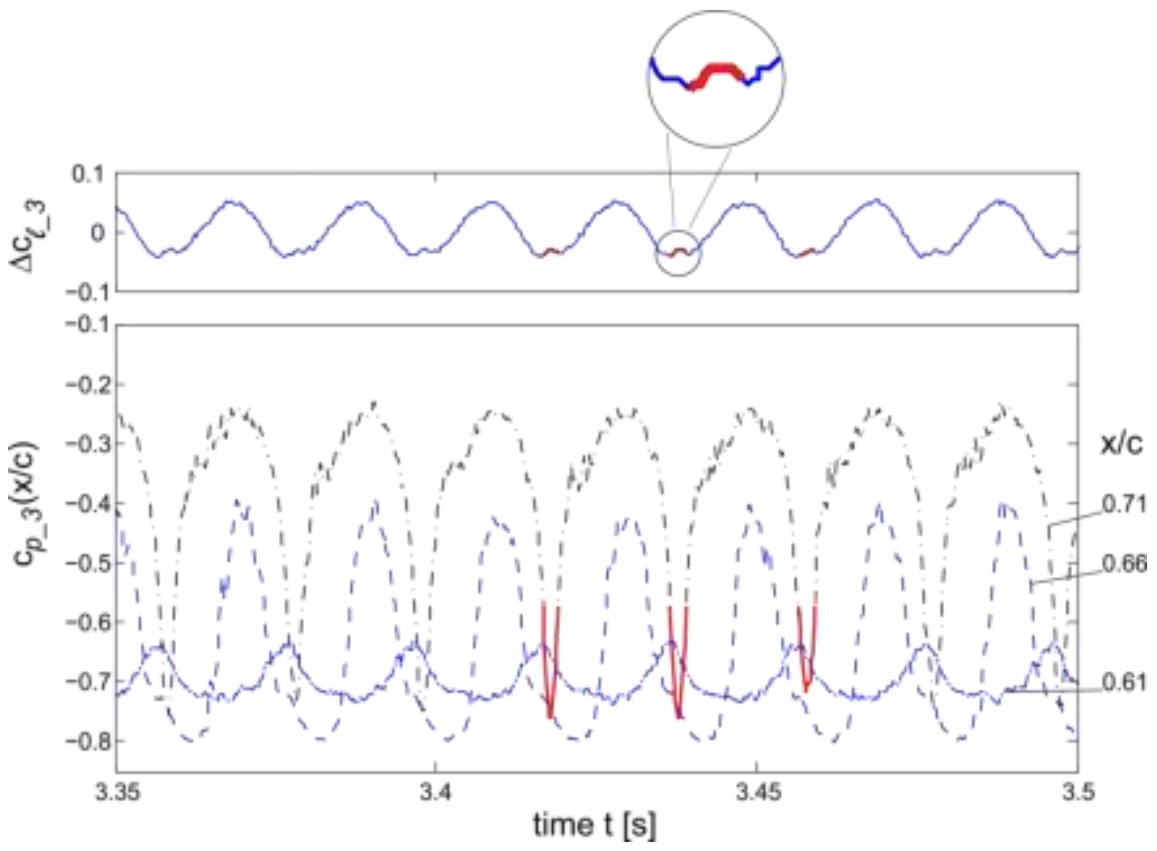

Figure 17. Time histories of three local pressures $\mathrm{c}_{\mathrm{p}_{3} 3}$ in the region of the second shock motion $(0.61<\mathrm{x} / \mathrm{c}<0.71)$. For comparison in the upper part there is again the time function of the sectional lift $\Delta \mathrm{c}_{13}$ from figure 15 .

$\Delta \mathrm{c}_{13}(\mathrm{t})$ as already shown in the preceding Figure 15 . Probably the wobble is also responsible for the very small relative maximum at $\Delta \mathrm{c}_{1 \_} \approx-0.042$, whose distance to the main maximum correspond roughly to the value of the peak-to-peak amplitude of the wobble.

In this context it should be noted that the signal of the displacement $h(t)$ is nearly harmonic, while the motion induced aerodynamic sectional lift forces $\Delta \mathrm{c}_{1 \_3}$ are asymmetric, reflecting significant nonlinear contributions. In order to find the causes for these nonlinear effects close inspection of the local pressures around the shock regions is suggested. 
Hence in Figure 17 the time histories of three local pressures $c_{p_{-} 3}$ in the region of the second shock motion $(0.61<\mathrm{x} / \mathrm{c}<0.71)$ are presented. For comparison in the upper part there is again the fluctuation of the sectional lift $\Delta \mathrm{c}_{13}$ from figure 15, which is the integral value of all pressures around the section. The locations of the individual pressures transducers $(\mathrm{x} / \mathrm{c}=$ $0.61,0.66$ and 0.71 ) become more clear when they are considered in the context of pressure distributions as presented in the preceding Figures 11 and 13 - in both figures the interesting part is marked. A little bit before (i.e. upstream) the second shock at $x / c=0.61$ the fluctuations are rather small compared with the both downstream cases. The form of the signal is rather asymmetric; the suction part is rounded while the pressure part appears spiky. In the shock regime at both neighboring downstream positions $\mathrm{x} / \mathrm{c}=0.66$ and 0.71 the fluctuations are very strong compared with the first one. In particular the form of the signal at 0.71 is very asymmetric and thus eye-catching. For this case the suction part is very spiky while the pressure part appears rounded. Apart from the amplitude both signals seem to be nearly out of phase. For clarity no more time histories are included in the Figure. However it should be complemented that beyond the shock and probably in partly separated flow the time function $\mathrm{c}_{\mathrm{p}_{-} 3}(\mathrm{x} / \mathrm{c}=0.76)$ has nearly the same asymmetric spiky form than at position $\mathrm{x} / \mathrm{c}=$ 0.71 , but the amplitude is significantly smaller. The form of the signal taken at the middle position $\mathrm{x} / \mathrm{c}=0.66$ is not as asymmetric as in both other cases and the phase-shift has values in-between. The sectional lift $\mathrm{c}_{13} 3(\mathrm{t})$ is the integral value of all local pressures around the section. Hence one can expect that the mentioned wobble is caused by one of the strong pressure fluctuations, occuring in the pressure signals of the shock region, which are shown in Figure 17. Indeed we found that mainly the eye-catching contribution of $\mathrm{c}_{\mathrm{p}_{-} 3}(\mathrm{x} / \mathrm{c}=0.71, \mathrm{t})$ is responsible for the wobble and the asymmetry in the sectional lift $\mathrm{c}_{13} 3(\mathrm{t})$. As demonstrated again in Figure 18 are displayed the total sectional lift $\mathrm{c}_{13}(\mathrm{t})$ and a modified total lift as dotted line, the modification means that the contribution $\mathrm{c}_{\mathrm{p}_{-} 3}(\mathrm{x} / \mathrm{c}=0.71, \mathrm{t}) \cdot \Delta \mathrm{x} / \mathrm{c}$ was omitted in the integration process. It is obvious that the wobble has disappeared and thus the asymmetry is now only small, an observation which is also confirmed in the PDF, which is not presented here. The peak to peak amplitude is significantly increased.

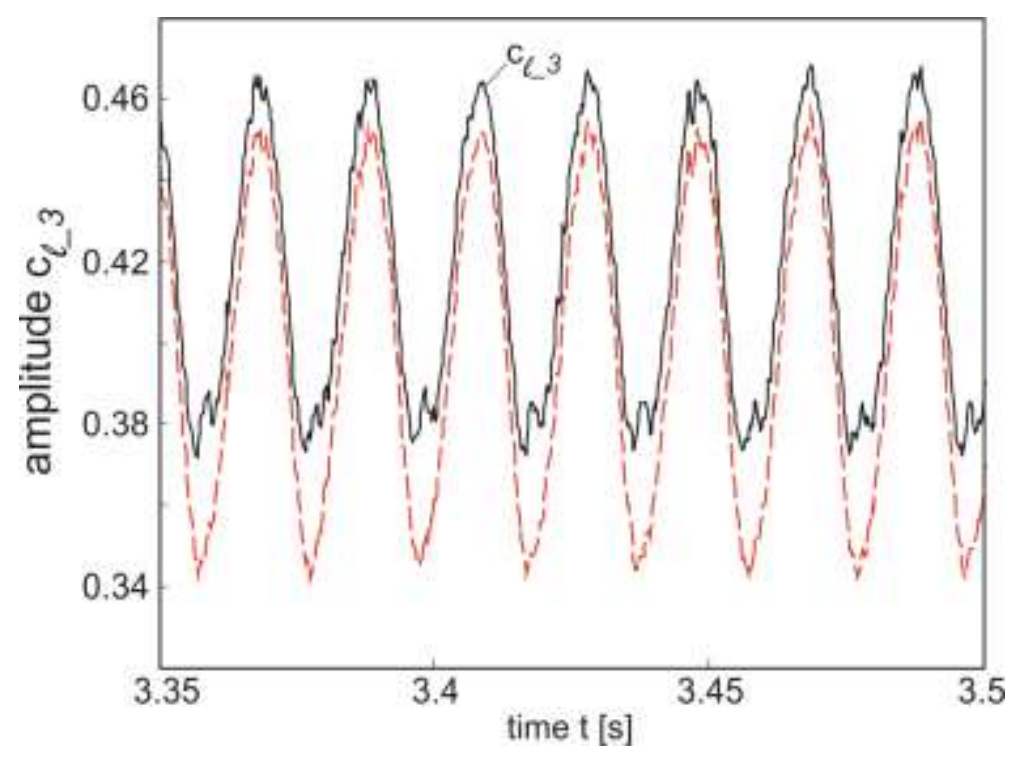

Figure 18. Sectional lift $\mathrm{c}_{1 \_3}(\mathrm{t})$ and a modified lift as dotted line, the modification means that the contribution of $\mathrm{c}_{\mathrm{p}_{-} 3}(\mathrm{x} / \mathrm{c}=0.71, \mathrm{t})$ was extracted. Here the mean lift is included. 
Schewe, Günter, and Mai, Holger. "Experiments on transonic limit-cycle-flutter of a flexible swept wing." Journal of Fluids and Structures 84 (2019): pp. 153-170. http://doi.org/10.1016/j.jfluidstructs.2018.07.005

Finally, in order to provide an impression of the deformation of the wing during the LCO test case in Figure 19 are plotted the normalized RMS-values of the individual accelerometers as

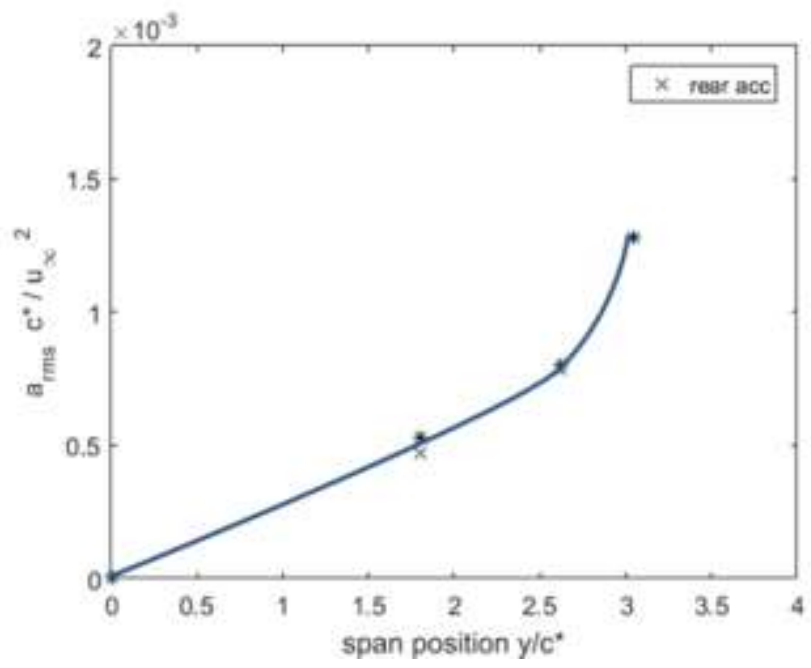

Figure 19. Normalized RMS-values of the individual accelerometers as function of the spanwise position $y / c^{*}$. The crosses are the results from both rear accelerometers.

function of the spanwise position $y / \mathrm{c}^{*}$. The analysis based on the cross spectral density showed that all signals are in phase thus the oscillation is a real mode and can be seen as onedegree of freedom motion. Although there are only a few points it is evident that in particular the outer wing is strongly bent.

\section{Discussion}

The behavior of the elastic wing at the measuring point MP "R 1520 MP 5" can be seen as the most significant part of our wind tunnel experiments and is thus the central test case. Already in Schewe (2013) it was demonstrated that the initial or general transient temporal behavior of self-excited flow induced vibrations can be described by a simple nonlinear model - the Landau equation. In its simplest form the Landau equation is a nonlinear differential equation for the envelope of the amplitude representing the global evolution of the system (Landau and Lifshitz, 1959, Manneville, 1990, 2004).

In Figure 20 is displayed the comparison between the measured flutter onset curve including the transition to a LCO and the envelope corresponding the Landau model. The Figure already shown in Schewe (2013) is presented also here as slightly modified version as it is an important part of the test case. The Figure shows the vertical displacement $h$ taken near the tip together with the associated time history of $\alpha$ at the root of the wing. At time $t=2.1 \mathrm{~s}$ there is the onset of self-excitation. Before the step in $\alpha$ there are also vibrations in the signal of low amplitude, which are excited by the turbulent fluctuations in the oncoming transonic flow. After the step of angle $\alpha$, self-excited oscillations set in exponentially and then there is a continuous transition to a LCO. We see that the envelope of the amplitude development is very well described by the solution of the Landau equation.

The input in the formula was solely the limit cycle amplitude $\mathrm{h}_{\mathrm{LC}}=0.009 \mathrm{~m}$, the time constant $\tau=0.293 \mathrm{~s}$. and the initial amplitude $\mathrm{A}_{0}=0.0006 \mathrm{~m}$. The time constant $\tau$, linked to damping $\delta$ and frequency $\mathrm{f}_{\mathrm{f}} *$ corresponding $\tau=-1 /\left(\delta \cdot \mathrm{f}_{\mathrm{f}} *\right)$ was determined at the onset of selfexcitation, where the grow- up of the oscillations is exponential. The matching process is objective, as $\mathrm{h}_{\mathrm{LC}}$ and $\tau$ are values, which can be directly obtained from the time function $\mathrm{h}(\mathrm{t})$. 
Only the matching of the initial amplitude $\mathrm{A}_{0}$ is somewhat subjective, as it shifts in the $\mathrm{t}$ direction.

Referring to the envelope it is a curve like the logistic function with exponential increase at small amplitudes and an exponential decay $(\sim 1-\exp (-t))$ when time $t$ becomes large i.e. the amplitudes approach the saturation value $\mathrm{h}_{\mathrm{LC}}$ - the limit cycle amplitude. That means the logistic function is point-symmetric around the turning point at the time, where the amplitude $h$ has reached half the limit cycle amplitude $h=h_{L C} / 2$. In Figure 20 the turning point is marked by a small circle. More details and the theoretical background are already described and discussed in Schewe (2013).

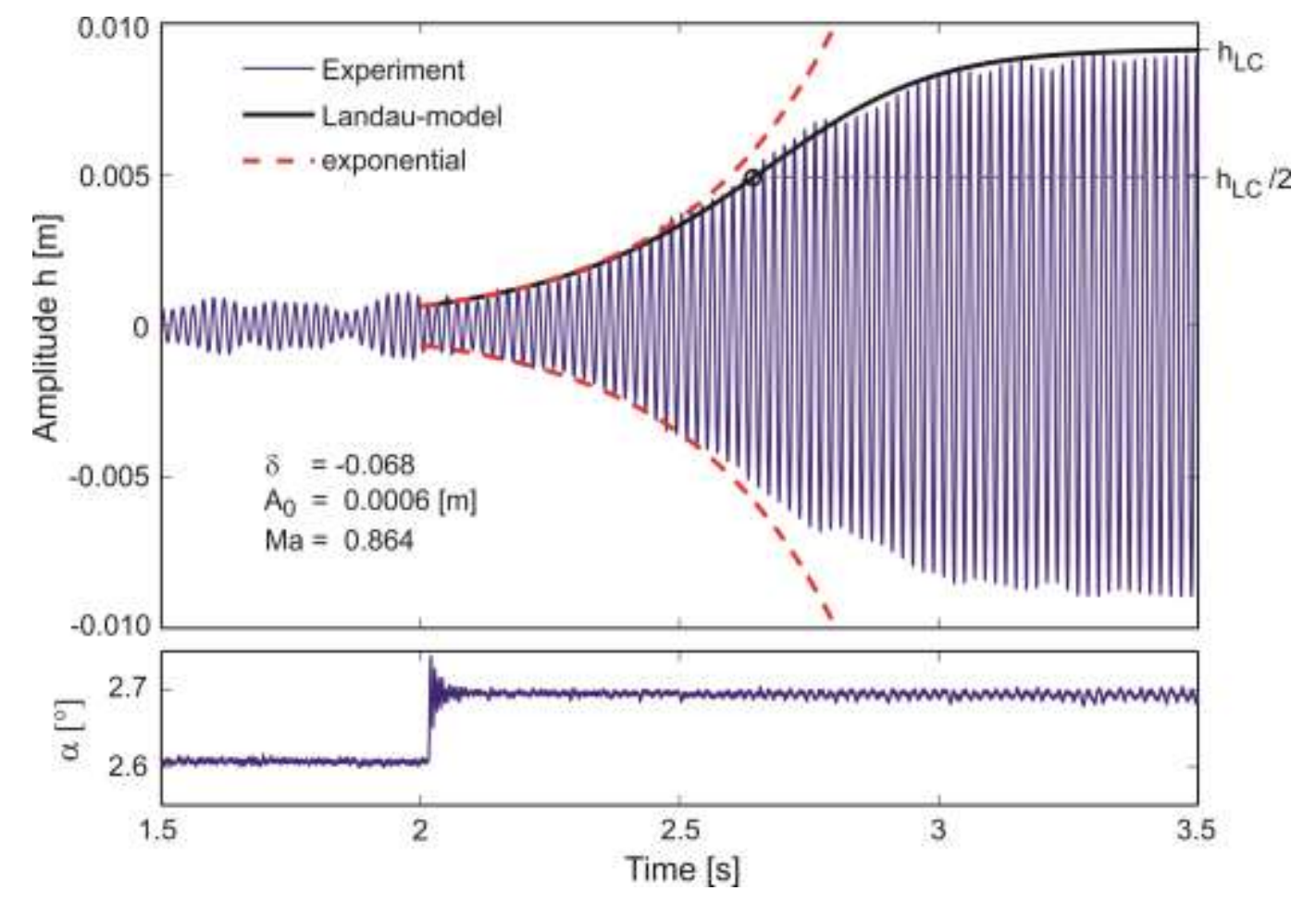

Figure 20. Time history of the vertical displacement $h$ taken near the tip. The onset of the self-excited oscillations was initiated by a small increment of the angle $\Delta \alpha$ (Figure from Schewe (2013); $\delta$ : damping, here negative i.e. built-up rate, $\mathrm{A}_{0}$ : initial amplitude)

Finally in the context of the test cases it is interesting to remark that in the same test campaign but a few days sooner a corresponding measurement was performed $[\mathrm{R} 1010 \mathrm{Mp}$ 6]. It was taken during the exploratory phase of the flutter tests applying nearly the same flow parameters and angle of attack $\alpha$ as in the measurement [R 1520 MP 5], described before. In general the question concerning reproducibility is a crucial point in experiments about bifurcation phenomena like the transition to flutter. The question arises, up to which degree the limit cycle amplitude can be reproduced. Nevertheless we can state that the mentioned limit cycle amplitude was nearly the same in both cases.

As noted in the introduction the last mentioned measurement was used by Stickan et al. (2014) as test case for their numerical simulations regarding the flutter phenomena. It was shown that in transonic flow the pressure distributions and in particular the double shock system could be simulated only if the structural model was able to simulate a small 
deformation of the wing section. The detailed structural shell model was also a condition for the simulation of the experimental LCO flutter test case.

In general the question regarding the ultimate causes for the amplitude limitation is one of the key-questions in "Transonic Flutter". The amplitude limitation is a nonlinear effect which is for example in the Landau equation reflected or modelled using a cubic term.

Stickan et al. calculated the local work at LCO cycle and it came out (Figure 19 of Stickan et al. (2014)) that mainly the shock motion in the outer wing is feeding energy in the wing structure thus exciting the oscillation. Regarding the limiter for the oscillation, the numerical simulation showed that the unsteady forces are nonlinear depending on the amplitude; in this case the forces are decreasing with increasing amplitude. The decrease of the forces in turn was found to be a consequence of strongly nonlinear motion of a weak oblique shock. In our experiments we also found that nonlinear behavior is obvious particularly in the sectional lift forces $\mathrm{c}_{1_{\_} 3}(\mathrm{t})$ of the outer wing-section.

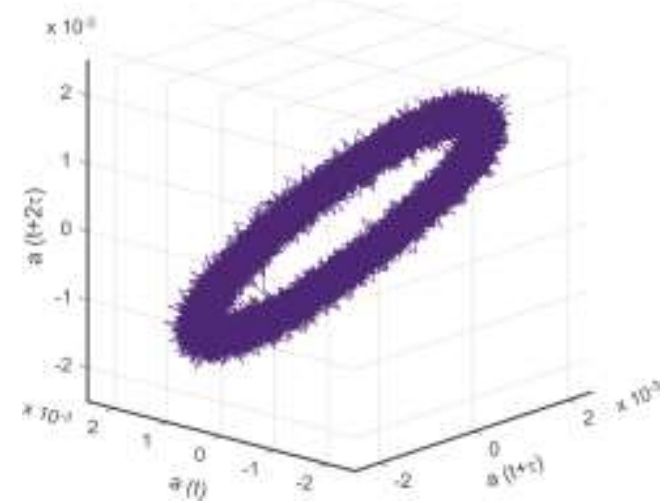

(a)

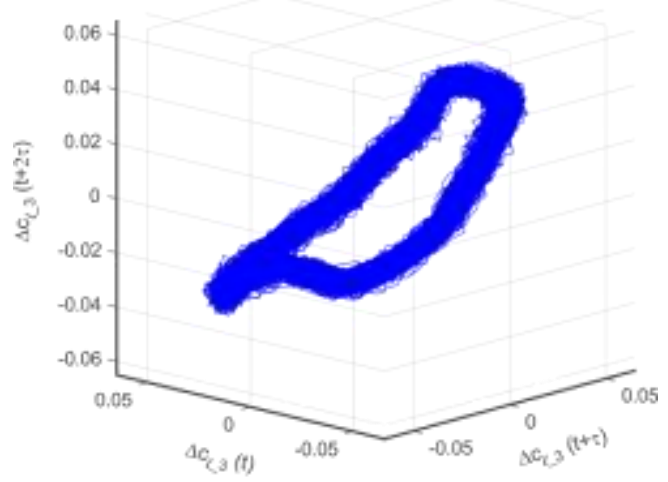

(b)

Figure 21. Three-dimensional phase diagrams, obtained by using time delay coordinates $\tau=\mathrm{T} / 4(\mathrm{~T}=1 /$ frequency of oscillation $)$.

a) Wing tip accelerometer b) sectional lift forces $\Delta \mathrm{c}_{1 \_3}$

The interrelation between the lift and the motion of the wing was displayed in Figure 15 and behaves as follows: both lift sectional forces $\Delta \mathrm{c}_{1 \_}(\mathrm{t})$ and $\Delta \mathrm{c}_{13}(\mathrm{t})$ and the displacement $\mathrm{h}(\mathrm{t})$ are out of phase. That means the lift is maximal at the bottom reversal point of the bending oscillation and vice versa. Further the displacement $h(t)$ is nearly harmonic (Figure 15) with a flatness of $F L=1.52$, while the motion induced aerodynamic sectional lift forces $\Delta \mathrm{c}_{1 \_3}(\mathrm{t})$ are asymmetric and thus having significant nonlinear contributions. The observation that the wing undergoes nearly harmonic motion is reflected in the three-dimensional phase diagram of the wing tip accelerometer displayed in Figure 21a. The significant nonlinear contributions in the motion induced forces are evident in a corresponding phase diagram for the sectional lift forces $\Delta \mathrm{c}_{1 \_3}(\mathrm{t})$, shown in Figure 21b. One reason for the nonlinearity is the small wobble, occurring around the bottom reversal point of the sectional lift $\Delta \mathrm{c}_{13} 3(\mathrm{t})$ as shown in the corresponding time function and probability density distribution. In this situation the second shock is most downstream. Close inspection of the local pressures showed that the eyecatching contribution of $\mathrm{c}_{\mathrm{p}_{-} 3}(\mathrm{x} / \mathrm{c}=0.71, \mathrm{t})$ is responsible for the wobble and the asymmetry in the sectional lift. Analysis of the time functions reveals that in the top reversal point there is a sharp suction peak in $\mathrm{c}_{\mathrm{p}_{-} 3}(\mathrm{t})$. Comparing this local pressure - in the same time window - with the integral lift $\Delta \mathrm{c}_{1 \_}(\mathrm{t})$, we see that the integrated value $\Delta \mathrm{c}_{1 \_3}$ goes through the negative half wave i.e. is minimal. However the mentioned suction peak creates a small but significant 
positive lift contribution and is thus responsible for the wobble in the integrated lift. By extracting the time history $\mathrm{c}_{\mathrm{p}_{-} 3}(\mathrm{t})$ from $\mathrm{c}_{1_{-} 3}(\mathrm{t})$ we have shown that indeed the contribution of $\mathrm{c}_{\mathrm{p}_{3} 3}(\mathrm{t})$ is causing the wobble in $\mathrm{c}_{13}(\mathrm{t})$.

So far we have discussed experimental results. The question arises: What are the consequences on the shock/boundary layer interaction and on the self-excitation process of the flutter oscillations. In Figure 22 there is a simplified sketch illustrating the shock motion and shock/boundary layer interaction during LCO. Both extreme cases are denoted by the

state

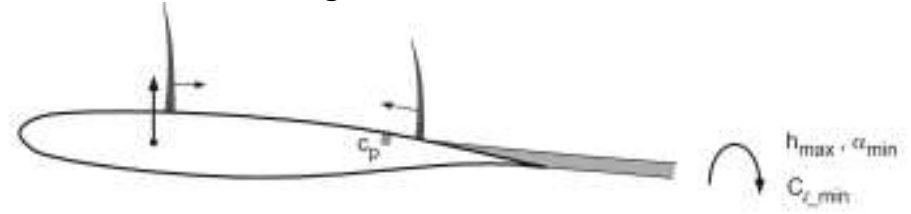

B
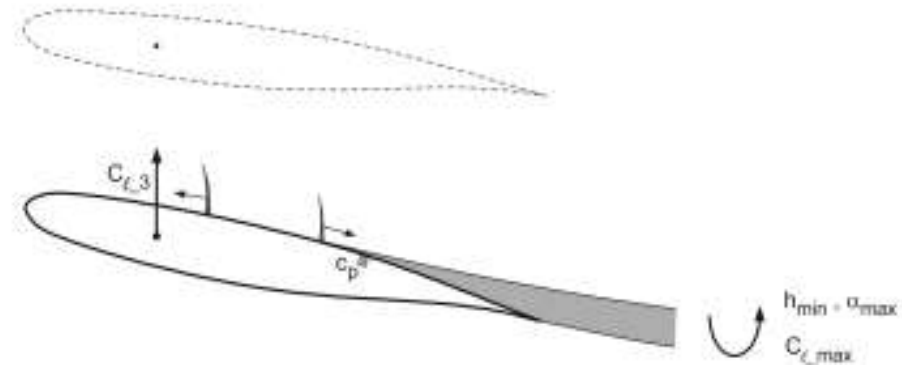

Figure 22. Simplified sketch of the shock motion and shock/boundary layer interaction during LCO. The states A and B represent the extreme situations occurring at a section of the outer wing. The filled square symbol shall represent the pressure transducer at the special position $\mathrm{x} / \mathrm{c}=0.71$, where the pressure signal is particularly eye-catching.

states $A$ at maximal bending $h_{\max }$ and state $B$ at the minimal value $h_{\min }$. During downstroke i.e. bending motion from state A to B the first shock is moving downstream (normal behavior) and the second shock travelling upstream. Thus the motion of the second shock is of type "inverse" with the following consequences: The boundary layer behind the shock is detached and there is a significant region of separated flow, whose extent is significantly increasing up to the extreme expansion at state $\mathrm{B}$. In the following upstroke motion from state $\mathrm{B}$ back to state A, the first shock is moving upstream and the second shock travelling downstream coupled with decreasing separation region. In both cases the second shock is traveling back and forth across the location of the pressure transducer at $\mathrm{x} / \mathrm{c}=0.71$. Considering the phenomena with reference from this special pressure location, during downstroke $(A \rightarrow B)$ the boundary layer detaches forming a separated flow region. During upstroke $(B \rightarrow A)$ however the separation breaks down leading to attached flow around the location of the pressure transducer.

The occurrence of the sharp suction peak in $\mathrm{c}_{\mathrm{p}_{-} 3}(\mathrm{t}, \mathrm{x} / \mathrm{c}=0.71)$ around the top reversal point of the bending oscillation $\mathrm{h}(\mathrm{t})$ is probably the consequence of the separation break-down during upstroke $(\mathrm{B} \rightarrow \mathrm{A})$. In other words, the break-down of separation leads to reattachment of the boundary layer around $\mathrm{x} / \mathrm{c}=0.71$. Thus the spiky suction peak and with that the wobble in $\mathrm{c}_{13}(\mathrm{t})$ is certainly a consequence of the reattachment process.

The phase shift obvious in the time histories of the three local pressures $c_{p_{3} 3}(t)$ in the region of the second shock motion (Figure 17, $0.61<\mathrm{x} / \mathrm{c}<0.71$ ) can be explained as follows: if there is a motion-induced transition between flow separation and reattachment as in the present case then usually the change between these two states is hysteretic. Typically the velocity of the shock motion is different for downstream or upstream direction. 
Schewe, Günter, and Mai, Holger. "Experiments on transonic limit-cycle-flutter of a flexible swept wing." Journal of Fluids and Structures 84 (2019): pp. 153-170. http://doi.org/10.1016/j.jfluidstructs.2018.07.005

It suggests itself that the hysteretic change between separation and attachment and the contribution of the wobble in the motion induced lift $\mathrm{c}_{13} 3(\mathrm{t})$ are significant sources of nonlinearity (Figure 21b) acting as a distortion during higher negative values of the exciting sectional lift. Consequently with increasing amplitudes a value will be reached where the aerodynamic excitation processes are balanced by the structural damping leading to a limit cycle oscillation.

Finally we can state that the interplay between the shock motion and the boundary layer separation plays an important role for the phenomena leading to the limitation of the oscillation amplitudes.

\section{Conclusions and outlook}

The investigation is concentrated on flutter experiments, which were analysed systematically in the transonic range for $0.84<\mathrm{Ma}<0.89$ and for six angles of attack from $1.46^{\circ}$ to $2.7^{\circ}$. Particularly the influence of flexibility and bending/torsion coupling was studied. Maps of stability and LCO amplitudes are presented. It was found that the unstable regions have the shape of islands, which can be approached from both sides i.e. also for decreasing tunnel pressure. This seems to be a special property of an elastic backward swept wing and caused particularly by a retwist of the outer wing.

For improving our knowledge about LCOs and for code validation purposes a LCO test case is presented containing the stage of built-up and the transition to the limit cycle.

The Analysis of the time dependent behavior shows that in the top reversal point of the flutter oscillation the location of the second shock is most downstream. At this time there is a sharp suction peak in the local pressure in the shock regime creating a wobble in the integrated lift consequently the motion induced aerodynamic sectional lift forces $\mathrm{c}_{1 \_3}$ are asymmetric and thus acting as amplitude limiter.

In general we can state that the interplay between the second shock motion and the boundary layer separation plays an important role for the phenomena leading to the limitation of the oscillation amplitudes.

Finally we can state that the present study and analysis was concentrated on the overall flutter behavior of the elastic swept wing, only one prominent flutter case was analyzed in-depth. For future work it would be interesting to investigate the onset of flutter oscillations in the vicinity of the boundary of stability in more detail and to do so at different locations.

Hence the question arises, which type of bifurcation occurs at which location. An answer is already in sight when considering figure 6 showing the limit cycle amplitude as a function of the stagnation pressure and the Mach number. The curves are looking like resonance curves, which are more or less bent leading to hysteresis ranges, particularly at the side of higher Mach numbers.

In general, a more detailed analysis of the underlying time histories, when an island of instability is crossed, would significantly increase our knowledge about the transonic flutter phenomena.

\section{Acknowledgement}

The authors would like to thank G. Dietz with whom we performed the experiments; his contribution is documented in Dietz et al. (2003). Further thanks go to W. Wegner and M. Braune for fruitful and stimulating discussions. Also the support of T. Gardner and Chr. Unger for helpful suggestions concerning the manuscript and the graphics is gratefully acknowledged. The investigation was financially supported by the Hermann von HelmholtzGemeinschaft (HGF) within the project "AEROSTABIL". 
Schewe, Günter, and Mai, Holger. "Experiments on transonic limit-cycle-flutter of a flexible swept wing." Journal of Fluids and Structures 84 (2019): pp. 153-170. http://doi.org/10.1016/j.jfluidstructs.2018.07.005 
Schewe, Günter, and Mai, Holger. "Experiments on transonic limit-cycle-flutter of a flexible swept wing." Journal of Fluids and Structures 84 (2019): pp. 153-170. http://doi.org/10.1016/j.jfluidstructs.2018.07.005

\section{References}

Arnold, J., Einarsson,G., Krüger,W. (2009) Multibody Simulation of an Aeroelastic Model. NAFEMS Int. Journal of CFD case studies, Vol 8. Dec

Bendiksen, O., O. (2008) Transonic limit cycle flutter of high-aspect-ratio swept wings. J. of Aircraft 45, pp. 1522-1533.

Bendiksen, O.,O. (2009) High-Altitude Limit Cycle Flutter of Transonic Wings J. of Aircraft 46, pp. 123-136

Bendiksen, O., O. (2011) Review of unsteady transonic aerodynamics: theory and applications. Progress in Aerospace Sciences 47.2: p. 135.

Dietz, G. Schewe, G. Kießling, F. Sinapius, M. (2003) Limit-Cycle-Oscillation Experiments at a Transport Aircraft Wing Model. Int. Forum Aeroelasticity and Structural Dynamics (IFASD) Amsterdam

Dietz, G. Schewe, G., Mai, H. (2006) Amplification and amplitude limitation of heave/pitch limit-cycle oscillations close to the transonic dip Journ. Fluids \& Structures 22 p. 505

Eckstrom, C. ,V., Seidel, D., A., Sandford, M., C. (1994) Measurement of unsteady pressure and structural response for an elastic supercritical wing. TP 3443, NASA,

Edwards, J. W., Schuster, D. M., Spain, C. ,V., Keller, D., F. and Moses, J.,W. (2001) MAVRIC flutter model transonic limit cycle oscillation test. TM 2001-210877, NASA

Jordan, P. (1946) Instationäre Vorgänge. in Monographien über Fortschritte der Luftfahrtforschung (seit 1939) ed. A. Betz, Band G Göttingen Monographs Concerning Progress in Germany Aeronautical Research (since 1939), short version also in chapter 4 of Meier, H., U. (2010)

Landau, L. D., Lifshitz, J.M. (1959) Course of Theoretical Physics (Vol. 6), Fluid Mechanics, Pergamon Press

Manneville, P. (2004) Instabilities, Chaos and Turbulence. Imperial College Press, London

Manneville, P. (1990) Dissipative Structures and Weak Turbulence. Perspectives in Physics, Academic Press

Meier, H., U. ed. (2010) German Development of the Swept Wing: 1935-1945. American Institute of Aeronautics and Astronautics, 2010. http://arc.aiaa.org/doi/book/10.2514/4.867552?mobileUi=0

Moses, G.,F., Pierce, D. (1977) The dynamic response of wings in torsion at high subsonic speed. CP 226, AGARD

Schewe, G., Deyhle, H. (1996) Experiments on Transonic Flutter of a Two-Dimensional 
Schewe, Günter, and Mai, Holger. "Experiments on transonic limit-cycle-flutter of a flexible swept wing."

Journal of Fluids and Structures 84 (2019): pp. 153-170. http://doi.org/10.1016/j.jfluidstructs.2018.07.005

Supercritical Wing with Emphasis on the Non-Linear Effects.

In: Proceedings of the Royal Aeronautical Society Conference on Unsteady

Aerodynamics, 17-18 July, London.

Schewe, G., Mai, H. and Dietz, G. (2003) Nonlinear Effects in Transonic Flutter with Emphasis on Manifestations of Limit Cycle Oscillations.

Journal of Fluids and Structures ,18, 3-22

Schewe, G. (2007) Force and Moment Measurements in Aerodynamics and Aeroelasticity using Piezoelectric Transducers.

Chapter 8.2 in Springer Handbook of Experimental Fluid Mechanics

Schewe, G. (2013) Flow-induced vibrations and the Landau equation. Journal of Fluids and Structures, 43, 256-270.

Schewe, G., Mai, H. (2018) Influence of Flexibility on the steady aeroelastic Behavior of a swept Wing in transonic Flow. Journal of Fluids and Structures, 81, 255-269.

Stickan, B., Dillinger, J., Schewe, G. (2014) Computational aeroelastic investigation of a transonic limit-cycle-oscillation experiment at a transport aircraft wing model. Journal of Fluids and Structures, 49, 223-241.

Tijdeman, H., R., Seebass, R. (1980) Transonic flow past oscillating airfoils. Annual Review of Fluid Mechanics 12.1: 181-222.

Zingel, H. et al. (1991) Measurement of steady and unsteady airloads on a stiffness scaled model of a modern transport aircraft wing.

Proc. Int. Forum Aeroelasticity and Structural Dynamics (IFASD)

DGLR 91-069, Aachen 